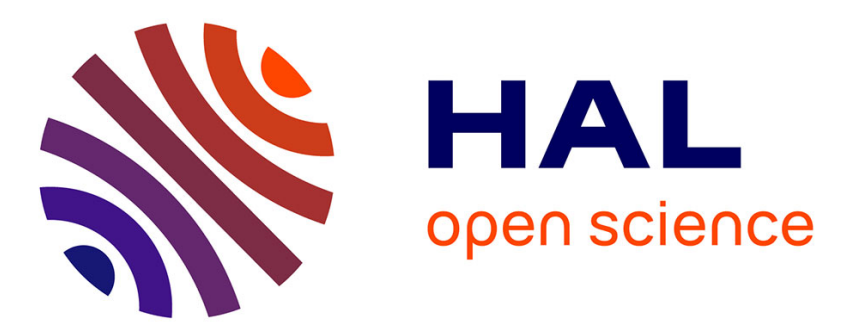

\title{
Le management de l'urgence dans les organisations ? Les réponses apportées par Napoléon Bonaparte
}

\author{
Laurent Mériade, Jean Marc Sales
}

\section{To cite this version:}

Laurent Mériade, Jean Marc Sales. Le management de l'urgence dans les organisations ? Les réponses apportées par Napoléon Bonaparte. Revue internationale de psychosociologie et de gestion des comportements organisationnels, 2020, XXVI (64), pp.165. 10.3917/rips1.064.0165 . hal-02934237

\section{HAL Id: hal-02934237 \\ https://hal.science/hal-02934237}

Submitted on 13 Nov 2020

HAL is a multi-disciplinary open access archive for the deposit and dissemination of scientific research documents, whether they are published or not. The documents may come from teaching and research institutions in France or abroad, or from public or private research centers.
L'archive ouverte pluridisciplinaire HAL, est destinée au dépôt et à la diffusion de documents scientifiques de niveau recherche, publiés ou non, émanant des établissements d'enseignement et de recherche français ou étrangers, des laboratoires publics ou privés. 


\title{
LE MANAGEMENT DE L'URGENCE DANS LES ORGANISATIONS ? LES REPONSES APPORTEES PAR NAPOLEON BONAPARTE
}

\author{
THE MANAGEMENT OF EMERGENCY IN ORGANIZATIONS? \\ THE ANSWERS PROVIDED BY NAPOLEON BONAPARTE
}

\author{
Laurent MÉRIADE ; Jean Marc SALES
}

\section{RESUME}

Dans la littérature, le management d'urgence (emergency management) a été essentiellement développé afin de répondre à des catastrophes. Il repose sur la définition et la mise en œuvre d'une organisation spécifique permettant de répondre à une situation de crise. Alors que l'urgence devient une situation de gestion permanente dans les organisations, il peut être intéressant de réfléchir à des modes de pensée et d'organisation en mesure d'y faire face de manière continue.

Comme il en existe pour les situations de crise ponctuelles, est-il possible de décrire certaines modalités d'un management (permanent) de l'urgence, applicables dans les organisations ?

Pour répondre à cette question de recherche, le management militaire se caractérisant souvent par un niveau d'urgence permanent, notamment en temps de guerre, nous tentons d'approcher, par une méthode historique, les modes d'organisation et d'administration que Napoléon Bonaparte adopte lors de ses principales campagnes militaires. La description et l'analyse managériale des évènements majeurs de ces campagnes nous permettent de mettre en lumière une certaine cohérence et une continuité de gestion, appliquées par Napoléon, qui peuvent inspirer les gestionnaires dans leur management quotidien de l'urgence.

Mots clés : urgence en gestion, management de l'urgence, pensée managériale, méthode historique, Napoléon

\begin{abstract}
In the literature, emergency management has been mainly built to respond to disasters. It is based on the definition and implementation of a specific organization to respond to a crisis or disaster situation. As emergency becomes a permanent management situation in organizations, it can be interesting to think about ways of thinking and organizing to deal with it on an ongoing basis.

As there are for specific crisis situations, is it possible to describe a (permanent) management of emergency applicable in organizations?

To answer this research question, since military management is often characterized by a permanent level of urgency, especially in times of war, we try to approach, by a historical method, the modes of organization and administration that Napoleon Bonaparte adopted during his main military campaigns. The description and managerial analysis of the major events of these campaigns allow us to highlight a certain coherence and continuity of management, applied by Napoleon, which can inspire managers in their daily emergency management.
\end{abstract}

Key words: management emergency, emergency management, managerial thinking, historical method, Napoleon 


\section{LE MANAGEMENT DE L'URGENCE DANS LES ORGANISATIONS ? LES REPONSES APPORTEES PAR NAPOLEON BONAPARTE}

\section{INTRODUCTION}

L'urgence et le débordement professionnel constituent des éléments du quotidien des managers (MINTZBERG, 1984), probablement encore plus prégnants aujourd'hui avec les accélérations induites par les technologies de l'information. L'urgence est devenue une contingence essentielle de l'environnement des entreprises (LAWRENCE ET LORSH, 1973) face à laquelle le management des organisations cherche perpétuellement de nouvelles réponses.

Le mot urgence vient du latin urgeo signifiant pressant, c'est à dire exercer une pression sur quelque chose ou sur quelqu'un à travers une action, une demande ou une attente consciente ou inconsciente. Urgeo donne aussi urgentia, qui veut dire nécessité pressante. Aussi, à la notion de pression se joint également celle de la nécessité de faire face à cette pression ou du moins de la prendre en considération. La sensation d'urgence naît de la pression et de la contrainte exercée par une situation, un contexte donné, ou bien encore par un individu ou un groupe d'individus sur un ou plusieurs autres individus (NOMBLOT ET THOMAS, 2009).

Dans les organisations, la gestion de l'urgence suppose la réalisation d'une action exceptionnelle et rapide pour empêcher un scénario défavorable de se dérouler (perdre un client, ne pas respecter un délai de réponse) ou pour remédier à une situation critique (perturber un process de production ou logistique) (JAUREGUIBERRY, 2003). Pour CANETTI et al. (2013) l'urgence en entreprise est une situation à risque réel dans un délai bref. Les « urgences » et leur mode de management dans les établissements hospitaliers sont une figure allégorique de 
ce besoin de réactivité face à une situation exceptionnelle à laquelle les managers et les personnels de toutes les organisations sont aujourd'hui régulièrement soumis. L'urgence se distingue de la crise qui est un moment de tensions extrêmes qui peut paralyser l'organisation. La crise peut effectivement créer une situation d'urgence mais pas systématiquement.

Le management est défini comme l'ensemble des dispositions qui permettent de mobiliser des ressources pour atteindre une fin (KEMPF, 2017). Face à l'urgence, le rôle du manager est de différencier ce qui est urgent ou ce qui constitue une priorité de ce qui est accessoire ou non urgent (ibid.). Sa mission est donc de distinguer l'important du superflu pour pouvoir agir. Par contre, il ne dispose que de peu d'outils pour hiérarchiser ces priorités. A la différence de la gestion de crises ou de catastrophes, quand il n'existe pas un management spécifique de l'urgence, le manager peut être seul face à ce défi. Dans beaucoup de situations d'entreprise, il ne fait appel qu'à son bon sens ou à son expérience pour déterminer ce qui relève de l'urgence et les actions et moyens qui peuvent répondre à celle-ci. Or, comme cela est réalisé pour la gestion ponctuelle de crises ou de catastrophes, il peut être intéressant d'explorer comment dans les organisations, à travers le management, il est possible de gérer l'urgence dans le travail quotidien des organisations.

Dans la littérature, le management d'urgence (emergency management) a été principalement développé afin de répondre à des catastrophes (catastrophes naturelles, accidents, actes terroristes, etc.) (WAUGH, 2015). Il repose sur la définition et la mise en œuvre d'une organisation spécifique permettant de répondre à une situation de crise en plusieurs étapes successives synthétisées notamment par le «cycle des catastrophes » et ses quatre phases (préparation, intervention, rétablissement et atténuation) (PHILIPS et al., 2016). Alors que l'urgence devient une situation de gestion permanente dans les organisations (AUBERT, 2003), il peut être intéressant de réfléchir à des modes de pensée et d'organisation en mesure d'y faire face de manière continue.

La problématique de notre article est donc la suivante : comme il en existe pour les situations de crise ponctuelles, est-il possible de décrire certaines modalités d'un management (permanent) de l'urgence, applicables dans les organisations?

Face à l'urgence permanente, les salariés et managers ont tendance à répondre par une activité permanente (ibid.). L'urgence succède alors à l'urgence, ce qui a pour effet de ne plus être en mesure de distinguer l'urgent de l'important ou l'accessoire de l'essentiel (ibid.). Ceci fait disparaitre la hiérarchie des priorités que le temps long imposait naturellement aux salariés ou aux managers dans les époques antérieures (AUBERT, 2006). 
Dans le domaine de la réflexion managériale, différents courants organisationnels (sociologie des organisations, théorie de la contingence, théories de la décision) ont intégré l'urgence dans leurs analyses mais sans construire, puisque ce n'était pas leur objet, une véritable réflexion globale sur sa gestion (RIVELINE, 1991).

Le management militaire se caractérise par un niveau d'urgence souvent permanent, notamment en temps de guerre, (tel que le suppose le vocabulaire employé : état d'urgence, intervention d'urgence, soutien d'urgence) qui impose des actions et des formes de management nécessitant probablement une continuité de pensées et d'actions managériales. A ce titre, les modes d'organisation et d'administration, que Napoléon Bonaparte adopte lors de ses principales campagnes militaires, semblent présenter une importante permanence et cohérence (DURHAM, 2015 ; SUCHET, 2004) dont l'analyse peut nourrir notre réflexion sur les manières de gérer l'urgence dans les entreprises. Notre objectif de recherche vise donc à déterminer, dans quelles mesures la gestion des campagnes napoléoniennes permet de circonscrire les contours d'un management spécifique de l'urgence applicable dans les organisations. Pour cela, nous revenons, à travers une analyse évènementielle des principaux faits saillants de ces campagnes, sur les préceptes et principes managériaux mis en œuvre par Napoléon Bonaparte pour faire face à des situations d'urgence.

Pour mener ce travail, notre article se structure de la manière suivante. Dans une première partie, nous effectuons une large revue de littérature du traitement de la question de l'urgence en gestion en distinguant au moins deux grands courants théoriques. Dans un deuxième temps, nous mettons en évidence la place déterminante que prend l'urgence dans les campagnes militaires menées par Napoléon que ce soit de manière imprévue, prévisible ou prévue. Dans une troisième partie, nous proposons une grille de lecture managériale des principaux évènements militaires de ces campagnes en mettant en avant les modalités du management de l'urgence appliquées par Napoléon. Enfin, nos principaux résultats mettent en avant, à travers la réflexion et les actions menées par Napoléon, le rôle déterminant de l'anticipation pour gérer l'urgence. En termes d'implications managériales, ceci définit des formes très illustrées de management de l'urgence potentiellement applicables dans les organisations.

\section{LE MANAGEMENT DE L'URGENCE DANS LA LITTERATURE}

Il y a encore 20 ans, le management de l'urgence était essentiellement connu des militaires : « un bon stratège repose sur ses capacités à agir en temps réel plus que sur ses aptitudes à planifier» (CANETTI et al., 2013). RIVELINE (op.cit.) constatait que «l'urgence est omniprésente dans la vie des affaires et totalement ignorée des théories des organisations » 
(p.82) En entreprise, le management de l'urgence apparait avec les nouvelles préoccupations managériales liées à la gestion des délais, à la tension des flux, à l'introduction de nouvelles technologies interactives. Mais, la structuration du champ scientifique relatif à ce management est encore à ses débuts et elle impose sans doute un développement conséquent des travaux qui s'y rapportent.

Pour répondre aux situations d'urgence dans les organisations, au moins deux courants de recherche en management les ont, tour à tour, intégrées dans leurs analyses afin de mesurer leurs effets directs et indirects sur le fonctionnement des organisations.

Dans le courant des «théories de l'entreprise » (LAWRENCE ET LORSH, op.cit. ; SIMON, 1957 ; COHEN et al, 1972), la théorie de la contingence définit nombre de contraintes et de situations qui limitent le champ d'action des acteurs des organisations. L'environnement de l'entreprise (BURNS ET STAKLER, 1994), les stratégies ou la structure de l'organisation (LAWRENCE ET LORSH, op.cit.), la taille de l'organisation (MINTZBERG, op.cit.), les technologies et progrès techniques utilisés (WOODWARD, 1980) sont dans la littérature des facteurs contingents communément mis en avant.

La théorie de la contingence d'inspiration anglo-saxonne ne fait réellement son apparition en Europe qu'au début des années 80 avec la propagation dans de nombreux pays de la crise provoquée notamment avec le second choc pétrolier (1978) (PIOTET et SAINSAULIEU, 1994). Les travaux doctoraux de REIX (1975) puis de de KALIKA (1984) ont les premiers illustrés l'apparition progressive des approches contingentes en France. La crise économique impose aux organisations d'adapter leurs structures d'organisation aux nouvelles pressions de leurs environnements. L'environnement des entreprises se complexifie par l'apparition de nouvelles menaces concurrentielles et financières. Dans son ouvrage «Managing » MINTZBERG (2009) en reprenant SAYLES (1989) mentionne combien la gestion est une activité de contingence, les gestionnaires agissent lorsque les routines s'effondrent et que des problèmes non résolus surgissent (p. 84). L'urgence, avec les nouveaux problèmes qu'elles créent, peut être rapproché de ces activités de contingence du management décrites par MINTZBERG (2009). Les managers doivent s'adapter à ces nouvelles contingences en construisant des structures souples et organiques, communicantes et participatives. Les manières dont les managers doivent répondre à ces nouvelles menaces contrastent avec les organisations bureaucratiques et simplifiées qui prévalaient au cours des périodes avec des environnements moins complexes.

L'urgence peut être considérée comme un nouveau facteur de contingence, moins illustré, mais de plus en plus visible dans les organisations. Par contre, dans ce courant 
théorique, elle n'est pas étudiée spécifiquement mais plutôt comme un facteur indirect provoqué par les autres facteurs définis ci-dessus (environnement, stratégie, technologie notamment). Aussi il parait intéressant de définir, aujourd'hui, sa place réelle dans l'environnement et le management des organisations.

La rhétorique sur le changement permanent et sur la nécessité de s'adapter à ces changements amplifient cette situation et amènent les organisations à proposer des solutions managériales pour répondre, en flux tendus, aux contraintes de leur environnement. Le juste à temps, dédié dans un premier temps plutôt aux activités industrielles et logistiques, devient peu à peu une modalité de gestion qui infuse l'ensemble des activités d'une organisation et impacte l'ensemble des personnels.

Management et urgence sont ainsi, de plus en plus reliées dans les organisations à travers un continuum récursif où la recherche d'une meilleure gestion amène souvent à l'urgence (AUBERT, 2003) qui à son tour impacte le management des organisations et ses représentations.

Pour nombre de théories de l'entreprise, les relations entre management et urgence renvoient aux problématiques de rationalité dans les organisations. Or, la rationalité limitée des décideurs, maintenant communément admise (SIMON, op.cit.), peut trouver encore de nouvelles limites dans l'urgence. Le modèle de la poubelle (COHEN et al., op.cit.) en révèle toute la substance. Prenant le cas des universités américaines, les auteurs démontrent que, face à l'urgence ou l'imprévision, les décisions peuvent devenir toujours plus irrationnelles (ibid.). L'urgence apparait dans les travaux de SIMON (1957) mais celui-ci la cite de manière très rapide pour signifier qu'il n'en traitera jamais tant celle-ci est un défi à la rationalité même limitée. Il la définit comme essentiellement subjective et particulièrement difficile à saisir car construite plus ou moins consciemment par les acteurs économiques pour justifier d'une action ou d'un mode opératoire (MATHEU et ROQUEPLO, 1991). En effet, tout peut être urgent quand il est apparu nécessaire d'agir ou de prendre une décision (ibid.). Pour les théories de l'entreprise, l'urgence est un défi permanent à la rationalité. Pour ces théories, chaque agent économique cherche à optimiser les critères sur lesquels il se considère jugé (BERRY et al., 1989). Le développement de l'urgence dans les organisations a aussi pour effet de réduire le nombre de critères pris en compte par chaque agent (ibid.). L'urgence tend à simplifier le modèle de décision autour de quelques éléments objectivables, ce qui va grandement perturber les capacités rationnelles du manager (MERIADE, 2016 ; 2017).

En effet, ce sont plus souvent les routines ou les habitudes qui, dans l'urgence, prennent le pas sur la créativité ou la réactivité pourtant si souvent mises à l'honneur dans le modèle très actuel 
d'entreprises libérée, innovante ou proactive. On peut dès lors s'interroger sur la capacité des managers et des organisations à gérer efficacement l'urgence tant il peut être délicat d'atteindre ses objectifs dans une situation temporelle toujours plus réduite.

Dans un deuxième courant plus «sociologique » (notamment issu de la sociologie des organisations), l'urgence dans les organisations constitue, au contraire, un assouplissement de l'environnement de travail qui rend de la liberté aux salariés et aux managers. Les «zones d'incertitude » décrites par CROZIER ET FRIEDBERG, (1977, p.46) peuvent s'agrandir au contact de l'urgence et offrir de nouvelles libertés aux salariés que ceux-ci peuvent mobiliser au profit d'une plus grande performance individuelle et organisationnelle. Si ces derniers auteurs ne citent pas explicitement l'urgence dans leur ouvrage, ils permettent de prendre en considération la temporalité comme un élément fondamental de l'environnement des organisations. En réduisant la complexité de l'organisation, l'urgence permet de développer la responsabilisation, l'autonomie et la créativité des personnels (MOISDON, 1990) qui, aujourd'hui, orientent de plus en plus les discours managériaux. L'urgence peut aussi être utilisée comme un moyen de compétition entre les entreprises qui en créant de l'urgence sur un marché peuvent faire disparaitre des concurrents (BONARELLI, 1990).

Ces deux courants de pensées ont donc contribué à définir deux formes d'intervention paradoxale de l'urgence dans les organisations. Face à cette ambidextrie de l'urgence dans la gestion des organisations, il est intéressant de se demander si certaines formes managériales ou organisationnelles peuvent apporter une réponse plus globale à la problématique du management de l'urgence qui, aujourd'hui, devient quasi permanente dans de nombreuses organisations.

En France, Les travaux du Centre de Gestion Scientifique (CGS) de l'Ecole des Mines de Paris ont permis de réaliser une synthèse des travaux de ces courants à travers une analyse axée spécifiquement sur l'urgence dans le cadre du management des organisations.

Dans les travaux fondateurs du CGS, RIVELINE (op.cit.) mentionne que pendant longtemps l'analyse du temps n'intervient pas dans les théories de l'organisation. Les entreprises sont censées adapter instantanément leurs productions et leurs prix à la demande et les clients sont parfaitement informés sur les produits et services présents sur un marché.

Les travaux du CGS ont commencé à construire, en France, une véritable conceptualisation de l'urgence en management BERRY et al., op.cit. ; MOISDON, op.cit. ; BONARELLI, op.cit.). Ils rattachent, dans un premier temps l'urgence à des circonstances exceptionnelles, par exemple dans les cabinets ministériels, de nombreuses décisions sont prises sous l'empire de la plus extrême urgence, ce qui sert l'intérêt général de manière somme toute acceptable 
(ROQUEPLO, 1990). Puis de manière plus approfondie RIVELINE (op.cit.), identifie deux types de complexité qui accompagne l'urgence dans le management des organisations. Tout d'abord la complexité de sens c'est-à-dire les différentes interprétations que l'on peut faire des situations d'urgence puis la complexité d'abondance c'est-à-dire la multitude d'informations qui permettent d'interpréter cette urgence.

FIXARI et PALLEZ (1990) décrivent l'urgence également sous l'aspect de ses effets bénéfiques pour l'organisation car elle et stimule la créativité, augmente l'implication des individus, de plus, elle simplifie la complexité décrite par RIVELINE (op.cit) et améliore l'efficacité plus grande. Jusqu'à présent les côtés négatifs étaient plutôt ressortis. Quoi qu'il en soit, il manque un cadre conceptuel.

Par la suite, pour illustrer la gestion de cette urgence, FIXARI et PALLEZ (1992) insistent sur l'importance du temps et du respect des délais c'est-à-dire des échéances. Ils décrivent le rôle moteur de la subjectivité des acteurs à la fois dans la perception de l'urgence mais aussi dans sa gestion. En fixant des échéances répondant aux situations d'urgence, les managers seraient en mesure à la fois d'anticiper mais aussi de hiérarchiser les situations d'urgence. Ici aussi, les travaux du CGS permettent de conceptualiser l'urgence en la distinguant d'autres concepts proches.

Ainsi, l'urgence et l'instantanéité ou la rapidité ne sont pas fondamentalement similaires (AUBERT, 2003). Au contraire, l'instantanéité crée de fausses situations d'urgence (ibid.) auxquelles on apporte des réponses rapides sans pour autant répondre à une situation d'urgence. L'instantanéité arrime l'individu à l'immédiat alors que l'urgence est beaucoup plus radicale (LAÏDI, 1999). Elle est une contrainte qui oblige à formuler une réponse, à mener une action (AUBERT, 2006) dont la survenance peut être plus ou moins rapide selon le type d'urgence. La rapidité en gestion correspond à la réactivité mesurée par la vitesse ou au temps de réaction d'une organisation aux changements inattendus de son environnement (BARZI, 2011). La réactivité vient de réagir (réagere en latin) qui consiste à opposer à une action, une action contraire afin d'annuler, neutraliser ou encore maitriser ses effets. La réactivité suppose donc qu'il y ait un changement et une rapidité d'action pour réagir (KIM et al., 2006 ; SPRALLS et al., 2010). Elle peut être une forme de gestion dans l'urgence mais elle n'est pas la seule. Quant à l'organisation réactive, elle est un état d'alerte permanent permettant de procéder aux ajustements jugés indispensables face aux changements de l'environnement de l'organisation (POIREL et al., 2015).

L'organisation face à l'urgence n'est pas non plus totalement une organisation agile qui est, elle, une organisation permettant de profiter d'un environnement marqué par des 
changements continus et imprévisibles (BREU et al., 2001 ; BARZI, 2011). YUSUF et al., (1999) décrivent plusieurs attributs de l'organisation agile (technologique, commercial, productif, humain, financier) qui répondent aux exigences des parties prenantes et de l'environnement de l'entreprise. Les concepts d'adaptabilité, de flexibilité et de réactivité sont au cœur du concept de l'agilité (SHARIFI et ZHANG, 1999). La flexibilité correspond au nombre d'alternatives futures, postérieures à une décision donnée (COHENDET et LLERENA, 1999 ; REIX, 1989) alors que l'adaptabilité est la capacité de l'organisation à ajuster ou modifier sa performance en fonction de la demande (KATAYAMA et BENNETT, 1999). L'organisation résiliente n'est pas non plus une structure entièrement tournée vers le management de l'urgence. Elle repose sur le principe de résilience qui consiste à savoir gérer l'imprévu (MOREL, 2018). La résilience s'oppose au principe de compliance (recourir aux règles de manière excessive). L'organisation résiliente est caractérisée par des règles frugales et de qualité, couplées à des relations humaines hautement fiables (Ibid.).

L'urgence présente donc ses propres spécificités, et, dans la littérature, le management d'urgence (emergency management) est la principale (voire la seule) forme managériale définie pour y répondre. Il vise à réagir à des catastrophes (catastrophes naturelles, accidents, actes terroristes, etc.) (WAUGH, 2015) pour lesquelles une organisation spécifique peut être mise en place de manière anticipée (interventions des secours, organisation des urgences hospitalières). Les catastrophes sont le résultat d'agents déclencheurs physiques ou humains et exacerbés avec des vulnérabilités environnementales ou organisationnelles (ibid.)

Les premiers travaux sur le management d'urgence ont eu pour objectif essentiel de déterminer comment les humains réagissent en cas de catastrophe. La première étude portée sur la collision de deux navires dans le port d'Halifax (6 décembre 1917) (PRINCE, 1920) a permis de construire un programme de recherche visant à identifier des comportements communs face à l'urgence pour définir un mode d'organisation face à l'urgence. La création en 1963, à l'Université de l'Ohio du Disaster Research Center a permis de structurer de manière très approfondie ce programme de recherche. Au cours de l'histoire la plus récente, les attentats terroristes du 11 septembre 2001 ont entraîné des changements organisationnels et programmatiques massifs dans la gestion des urgences (HADDOW ET AL., 2017).

Pour faire face aux risques de survenance de ces phénomènes d'urgence connus mais aléatoires, le management d'urgence s'appuie sur les quatre phases du « cycle des catastrophes » (PHILIPS et al., 2016) (c.-à-d. la préparation, l'intervention, le rétablissement et l'atténuation) afin de gérer la survenance d'événements majeurs. CAO et al. (2018) définissent les systèmes de management d'urgence à partir de cinq invariants qui permettent de les caractériser : un système 
d'information propre à un risque connu, un système de commandement et de coordination des activités, une organisation logistique dédiée, une organisation règlementaire et législative permettant d'intervenir en urgence. Ils sont mis en place dans des situations spécifiques et ponctuelles pour faire face à des phénomènes connus.

Le management de l'urgence telle qu'elle apparait aujourd'hui dans les organisations, par son caractère à la fois permanent et transversal, ne dispose pas de dispositifs totalement dédiés à sa mise œuvre comme cela peut être le cas pour des situations de crise ou de catastrophe. Même si elle peut faire appel à certains éléments des systèmes de management d'urgence tels que nous les connaissons, il est encore difficile de cerner précisément la manière dont ces éléments s'articulent et forment un tout cohérent pour gérer l'urgence. Il apparait important de réfléchir aux manières dont l'urgence peut être gérer de manière globale et permanente dans les organisations. Il apparait que l'histoire militaire dispose d'un certain nombre d'exemples de campagnes militaires héroïques, au long cours (celles d'Alexandre le Grand, de Charlemagne, ou encore de Napoléon Bonaparte), présentant, dans le temps, au-delà des modifications apportées par la sophistication du matériel de guerre et les innovations militaires, une certaine continuité et cohérence sur le plan stratégique et organisationnel qu'il peut être intéressant d'analyser pour apporter des réponses à cette interrogation. Dans son ouvrage Simply Managing, MINTZBERG (2013), mentionne combien le management n'est pas une science. Pour MINTZBERG (op.cit.), la gestion applique certainement la science (les gestionnaires doivent utiliser toutes les connaissances qu'ils peuvent acquérir) mais une gestion efficace dépend davantage de l'art et s'enracine surtout dans l'artisanat (p.8). L'art produit des idées et des visions fondées sur l'intuition et l'artisanat permet d'apprendre de l'expérience - en travaillant les choses au fur et à mesure que le gestionnaire progresse. Ce management et la pensée managériale qui s'y rapporte est assez caractéristique du mode de fonctionnement de Napoléon dans sa gestion de l'urgence. Napoléon n'est en aucun cas dogmatique, il s'adapte, tel un artisan, à l'environnement auquel il fait face. La description et l'analyse de ses modes de management de l'urgence peuvent apporter des éclairages complémentaires aux travaux portant spécifiquement sur la gestion de l'urgence dans les organisations (notamment ceux du CGS). L'évidence de l'urgence qui renvoie au vécu quotidien des managers a souvent limité les travaux portant sur l'étude spécifique de sa gestion (FIXARI ET PALLEZ, 1992, p.78). Pourtant l'urgence est souvent très révélatrice du fonctionnement profond des organisations (ibid., p.86) mais son analyse impose de porter une attention soutenue et singulière aux relations qu'elle entretient avec l'ensemble des dispositifs managériaux des organisations. C'est ce que nous proposons de réaliser en étudiant de manière spécifique les articulations qui s'opèrent 
entre la pensée managériale de Napoléon et les situations d'urgence auxquelles il doit régulièrement faire face.

\section{UNE METHODE HISTORIQUE APPLIQUEE AUX SCIENCES DE GESTION}

Dans l'histoire de la business history, CHANDLER (1992) a grandement inspiré les approches scientifiques vouées à l'utilisation de l'analyse des faits ou évènements historiques pour contribuer à une compréhension plus générale des mécanismes et des logiques du fonctionnement des organisations dans leur ensemble. En France, la Business History s'est développée progressivement dans les années 1970 à un rythme plus irrégulier qu'aux Etats Unis $\mathrm{du}$ fait de l'absence partielle dans les entreprises d'une importante conciliation entre rétrospective et modernité. Ce sont plutôt les entreprises publiques (EDF, France Telecom, SNCF, La Poste) qui ont joué un rôle important dans le classement et la diffusion des archives d'entreprise permettant de construire un dialogue entre gestionnaires, historiens et, aujourd'hui, chercheurs en gestion.

L'étude de cas historique est particulièrement mis lumière par les travaux de CHANDLER (ibid.) afin, dans l'esprit de l'Ecole des Annales, de reconstituer l'histoire telle qu'elle s'est faite (SEIFFERT et GODELIER, 2008). Même s'il ne cite pas précisément cette école dans ses ouvrages, l'esprit de l'école des Annales de Marc Bloch souffle sur le travail de CHANDLER (ibid.). Ce dernier et ses disciples, dans leurs travaux ne cherchent effectivement pas à accumuler des faits pour faire une histoire « historisante » mais, au contraire, il essaie de reconstituer l'histoire telle qu'elle s'est faite pour comprendre voire proposer une description des modes et règles de fonctionnement des entreprises les plus significatives (ibid.). Si nous nous inscrivons dans cette tradition historiographique en gestion, nous opérons, dans ce travail, une analyse, quelque peu inverse, qui vise plutôt à mobiliser les évènements historiques pour illustrer la pensée et l'action managériales. Pour cela, les travaux historiques en marketing et en gestion (SAVITT, 1980 puis SMITH et LUX, 1993) ont «méthodologisé » une approche évènementielle (figure 1) en deux temps méthodologiques (devis de recherche et analyse historique) et cinq étapes principales (mise en forme de la problématique, procédures de recherche, investigation, synthèse et interprétation) 
Figure 1. La méthode historique (Smith et Lux, 1993, d'après Savitt, 1980)

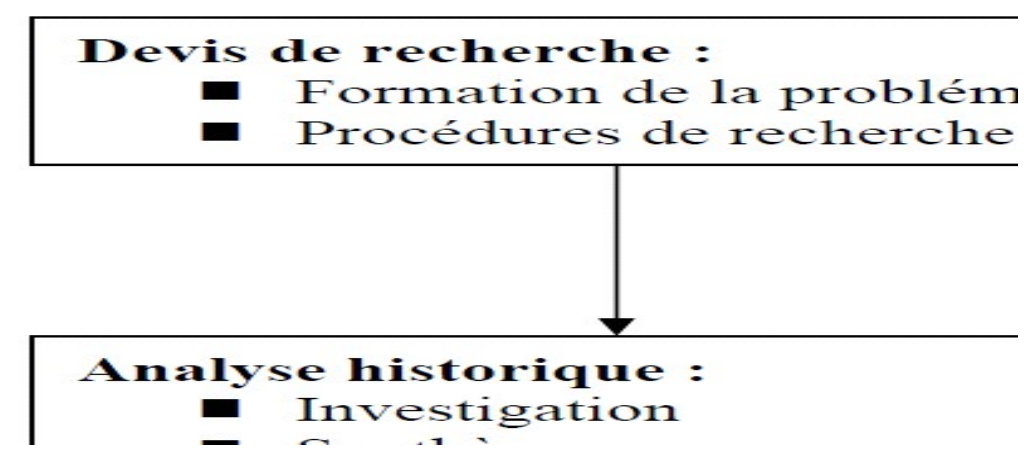

Le choix de cette approche « rationaliste » est quelque peu dicté par notre volonté de mobiliser les nombreux travaux historiques existants sur Napoléon et ses campagnes militaires plutôt de relater et reconstituer l'histoire telle qu'elle s'est faite. C'est pour cela que nous nous sommes concentrés sur l'analyse des travaux historiques les plus robustes traitant de ce sujet afin de commencer à étudier les éléments descriptifs du management de l'urgence que peut opérer Napoléon au cours de ses campagnes. L'étude des archives relatives à ces campagnes ne nous est pas apparue impérative notamment parce que les travaux existants nous paraissaient, dans un premier temps, particulièrement riches et pertinents à exploiter. Il est probable, que dans de futurs travaux sur cette période historique, cette recherche devra être approfondie par une analyse complémentaire d'archives de ce premier travail exploratoire.

Dans un premier temps, en mobilisant cette approche, nous avons construit une problématique (celle du management de l'urgence dans les organisations) à partir de notre perception et notre connaissance de l'histoire napoléonienne. Cette première étape doit nous permettre d'appréhender au mieux la perpétuelle subjectivité du chercheur en histoire (SAVITT, op.cit.; BOUSQUET, 2005) afin de l'anticiper lors des étapes suivantes et notamment celles de synthèse et d'interprétation. Pour assurer la validité externe de nos données, nous avons construit une procédure de recherche à partir d'une investigation approfondie de la littérature permettant de sélectionner les sources, documents, et preuves historiques permettant d'approcher notre problématique par la littérature historique napoléonienne. La validité interne étant obtenu par la « qualité » du support et de l'information retenu (Bousquet, op.cit.). A partir de notre connaissance et nos analyses antérieures de l'histoire de la période, nous avons retenu les ouvrages et écrits généralement les plus cités dans les travaux traitant des évènements et de l'organisation militaire napoléonienne sous le Directoire (1795 - 1799), le Consulat et l'Empire (1799 - 1815) (BOUDON, 2000 ; 
CASTELOT, 1967 ; WIEVIORKA et al., 2018; MIQUEL, 2005 ; PIGEARD, 2004, TRANIE, 1999, COLSON, 2011, TULARD, 2012).

Ceci nous a permis, dans un deuxième temps, de construire une analyse historique en réalisant des allers-retours réguliers entre les résultats de notre revue de littérature sur le management de l'urgence dans les organisations et nos supports et documents de recherche historiques.

\section{NAPOLEON: UNE PENSEE TOURNEE VERS LE MANAGEMENT DE L'URGENCE}

Pour Napoléon, faire face aux pressions immédiates de manière efficace -tant sur les plans externe, qu'interne- n'est compréhensible que si l'on se place sur les plans psychologique et intellectuel de son construit personnel. Napoléon est un être qui s'est bâtit dans la douleur. De culture Corse -laquelle a été rattachée à la France par le traité de Versailles un an avant sa naissance- il est éloigné de la cellule familiale dès sa $9^{\text {ème }}$ année par son entrée au Collège d'Autun puis à l'école militaire de Brienne. Il la quittera 5 ans après pour intégrer l'école militaire supérieure de Paris de 1784 à 1785. Son goût pour les mathématiques le dirige tout naturellement vers l'artillerie, arme à laquelle il est affecté dans le cadre du régiment de la Fère en garnison à Valence (1785 - 1791). Capitaine d'artillerie, il contribue au siège victorieux de Toulon en 1793. Désormais connu et apprécié par le clan Robespierre, il devient Général de brigade en 1794 et manque de peu de les suivre dans leur chute. Barras, va le relancer en lui demandant de réprimer une insurrection royaliste, ce qu'il fait le 13 vendémiaire an IV (5 octobre 1795). Devenu membre du Directoire, son nouveau protecteur lui présente Joséphine sa future épouse, puis lui confie le commandement de l'armée d'Italie. C'est le début de l'épopée.

Mais, au-delà de la personne de Napoléon, l'urgence apparait dans l'action militaire de manière assez systématique (COLSON, 1999). Napoléon doit faire face à l'urgence des coalitions des pays ligués contre lui. De 1792 à 1815, elles seront au nombre de sept. Dès son arrivée au pouvoir en 1799, les offres de paix de Bonaparte sont repoussées par Londres et Vienne qui voient dans le $1^{\mathrm{er}}$ consul le continuateur de la révolution. Bonaparte devra donc faire face et se défendre continuellement des attitudes hostiles d'importants pays européens (Angleterre, Autriche, Russie, Prusse pour ne citer que les principaux). Exercice d'autant plus difficile que son pouvoir est fortement contesté au début du Consulat notamment par les royalistes. Aussi, les exemples de situation d'urgence sont nombreux dans l'épopée Napoléonienne (perte de la flotte française à Aboukir en 1798, nécessité de vaincre rapidement lors de la $2^{\text {ème }}$ campagne d'Italie et à Austerlitz avant l'arrivée des Prussiens, embossage de 
Villeneuve à Cadix en 1804 empêchant l'invasion de l'Angleterre ce qui provoque la pirouette de Boulogne, émeutes de Madrid de 1808, etc.). Napoléon y fait face par des principes et actions qui présentent une certaine continuité et qu'il nous semble très opportun d'analyser (SUCHET, op.cit.).

Les deux grands axiomes de sa pensée managériale se rattachent à son leadership ainsi qu'aux principes militaires qu'il applique avec rigueur. Ces axiomes permettent à Napoléon Bonaparte d'être dans l'anticipation, véritable catalyseur qui déclenche son action sur les plans de la communication et de l'organisation. Les résultats en termes de cohésion et de concentration des forces sont ainsi particulièrement efficaces pour faire face à l'urgence et la gérer.

\subsection{Leadership et style de management}

Tout d'abord, dans sa manière de mener et gérer les hommes, à partir de compétences acquises ou de circonstances imposées par les événements, Napoléon déploie un management assez proche de certains styles décrits par LIKERT (1967). Ce dernier auteur a consacré la majeure partie de son énergie et de son temps à conseiller et à maintenir des entreprises, des organisations et services publics (KISH, 1982). Il semble étonnant qu'il ait trouvé, à côté d'innombrables apparitions publiques, le temps pour écrire plus de cent articles et trois livres : New Patterns of Management (1961), The Human Organization : its Management and Value (1967) et New Ways of Managing Conflict (1976), le dernier en collaboration avec Jane Gibson Likert. Ces ouvrages, basés sur des enquêtes et des études dans les milieux d'affaires, industriels et gouvernementaux, traitent de la gestion participative et ils ont progressivement structuré la description de ses quatre styles de management (autoritaire, paternaliste, consultatif et participatif) (LIKERT, op.cit.; KISH, op.cit.).

Napoléon est un leader charismatique. Max WEBER (1946) définit le leadership charismatique comme « reposant sur la dévotion à la sainteté exceptionnelle, à l'héroïsme ou au tempérament exemplaire d'une personne individuelle, et des modèles ou de l'ordre découvert ou ordonné par lui ». Il perçoit le charisme comme « une certaine qualité d'une personnalité individuelle, en vertu de laquelle il est à part indépendamment des hommes ordinaires et traité comme doté de puissances ou de qualités surnaturelles, surhumaines spécifiquement exceptionnelles. Celles-ci sont telles qu'elles ne sont pas accessibles à la personne ordinaire, mais sont considérées comme d'origine divine ou comme exemplaires, et basé sur elles, l'intéressé est traité comme un leader » (ibid.).

Les propos et l'histoire de Napoléon illustrent cette définition, point par point, à travers quelques exemples très significatifs de son histoire personnelle et militaire. Ainsi, Napoléon 
construit son image de sainteté notamment par la création de la Saint Napoléon le 15 août, et la vénération de ses soldats (BOUDON, 2000). Son héroïsme et son comportement exemplaire sont symbolisés, par exemple, par le passage du pont d'Arcole un drapeau à la main (même si cela a été exagéré par la propagande Napoléonienne). Par ailleurs, le modèle Napoléonien s'est construit par la lente maturation, opérée par le futur Empereur, pour passer du Consulat à l'Empire. Napoléon réussit à incarner celui qui met fin à la Révolution (« Ni bonnet rouge, ni talon blanc je suis national ») et qui passe à l'Empire, et à son statut d'Empereur, tout en restant garant des principes de ladite révolution (Egalité, reconnaissance des mérites...). « Je jure de maintenir l'intégrité du territoire de la République ; de respecter et de faire respecter les lois du concordat et la liberté des cultes ; de respecter et faire respecter l'égalité des droits, la liberté politique et civile, [...]». (Napoléon durant la cérémonie du sacre du 2 décembre 1804).

Concernant les qualités d'une personne individuelle à part, indépendamment des hommes ordinaires, définis par Weber, très tôt, dès son entrée à Brienne (de mai 1779 à octobre 1784), Napoléon se singularise. Il est à part de ses condisciples (exceptés Des Mazis et Bourrienne qui deviendra son secrétaire particulier). Enfin, par ses références à ses pouvoirs divins ( «Souvenez-vous que je marche accompagné du dieu de la guerre et du dieu de la fortune »Conseil des Anciens, 19 brumaire an VIII - 10 novembre 1799), Napoléon construit son image de leader charismatique disposant de qualités surnaturelles, surhumaines comme d'origine divine (18/19 Brumaire cité par CASTELOT, 1967).

Dès lors, à partir de son leadership charismatique, le management de Napoléon peut être décrit comment balançant entre :

- le style autoritaire / directif (LIKERT, op.cit.) qui renvoie à l'urgence des situations, - le style persuasif (ibid.) à même de créer la cohésion de l'armée au besoin dans l'urgence, style qualifié aussi de paternaliste (tableau 1).

Ce style de management associé au charisme du leader, semble être un élément fondateur du management des urgences auxquelles Napoléon doit faire face au cours de ses campagnes militaires.

Dans son ouvrage «Le gouvernement participatif de l'entreprise », Likert (1974) expose son formulaire d'enquête auprès des managers (« Tableau 3.1 des caractéristiques d'organisation et de performance des divers systèmes de gestion fondées sur une analyse comparative » p. 13 et suivantes). Pour illustrer nos propos, nous nous sommes appuyés à partir du tableau 1 sur les caractéristiques opérationnelles repérées par Likert dans son ouvrage (degré de communication et d'interaction en vue de la poursuite des objectifs à atteindre, communication descendantes, processus d'interaction et d'influence, influence des subordonnés sur les objectifs, les méthodes 
et les activités de leurs services et de leurs divisions, intérêt psychologique des supérieurs pour leurs subordonnés). Cela nous permet d'illustrer avec plus d'exactitude les deux systèmes de management adoptés par Napoléon.

Tableau 1. Le style de management Napoléonien selon les typologies de Likert (1967)

\begin{tabular}{|c|c|}
\hline $\begin{array}{l}\text { autoritaire / directif } \\
\text { (Système 1) }\end{array}$ & $\begin{array}{l}\text { Style particulièrement efficace en situation d'urgence } \\
\text { «Le général qui voit avec les yeux des autres n'est pas capable de commander une } \\
\text { armée » } \\
\text { Les maréchaux et généraux n'ont pas d'influence sur les objectifs, les méthodes et } \\
\text { les activités de leurs services et de leurs divisions. La « grande tactique » autrement } \\
\text { dit la stratégie est du ressort de Napoléon. }\end{array}$ \\
\hline \multirow{2}{*}{$\begin{array}{l}\text { Paternaliste, } \\
\text { Persuasif } \\
\text { (Système 2) }\end{array}$} & $\begin{array}{l}\text { Napoléon est certes directif, il tranche mais il est à l'écoute des besoins de ses } \\
\text { soldats : prise en compte des blessés (le soldat français est en Europe le mieux } \\
\text { soigné). "Sachez écouter, et soyez sûr que le silence produit souvent le même effet } \\
\text { que la science." (Napoléon Bonaparte - Instructions pour le prince Eugène). } \\
\text { L'association de l'écoute et la décision directive est également un élément essentiel } \\
\text { de sa capacité à prendre les bonnes décisions dans des moments d'urgence. }\end{array}$ \\
\hline & $\begin{array}{l}\text { Il explique et cherche à faire adhérer: } \\
\text { - aux valeurs de la république «La première des vertus est le dévouement à la } \\
\text { patrie. » } \\
\text { - à l'ordre qu'il construit à travers notamment les distinctions telles que la Légion } \\
\text { d'honneur « On ne conduit le peuple qu'en lui montrant un avenir : un chef est un } \\
\text { marchand d'espérance. » } \\
\text { Cet aspect de son management est favorable à la cohésion de ses troupes qui elle } \\
\text { aussi, comme on le verra plus loin permet de répondre efficacement aux situations } \\
\text { d'urgence. } \\
\text { Les officiers sont incités à s'intéresser vraiment au soldat et même pour un chef de } \\
\text { bataillon à connaitre leur nom ! Exemple du rôle du Colonel dans l'armée } \\
\text { Napoléonienne : il doit motiver les troupes, gérer la diversité et les conflits internes }\end{array}$ \\
\hline
\end{tabular}

En ce qui concerne l'organisation Napoléonienne, il semble impossible de tendre vers le système 4 (participatif) pour des raisons :

- liées aux ressources tout d'abord car Napoléon ne dispose pas des ressources humaines nécessaires en terme de compétences (insuffisance d'officiers et de sous-officiers due aux pertes mais aussi à l'absence d'école de formation spécifique d'officier d'EtatMajor, etc.)

- psychologiques, car historiquement les mentalités de l'époque intègrent mal le notions de délégation et Napoléon par son caractère entre en partie dans ce mode de pensée

- militaires l'essence même de la guerre en créant des situation d'urgence nécessite prioritairement l'emploi des systèmes 1 et 2 .

- organisationnelles. La structure conçue par Napoléon permet l'application du principe de concentration des forces ce qui nécessite la dispersion, la réunion et la concentration des forces. Cela est peu compatible avec un système délégatif.

Cependant, la pensée managériale de Napoléon semble respecter un certain « continuum » entre les systèmes 1 et 2, mais aussi 3 (consultatif). En effet, pour ce dernier style, Likert définit la 
communication descendante « sans exclure certaines initiatives prises à des niveaux inférieurs » (p. 16). Ainsi les ordres stratégiques sont pris par Napoléon de manière centralisée. Cependant, il préconise aussi de les formuler de manière précise mais large de manière à ce que les responsables des corps d'armées agissent de manière autonome dans ce cadre tout en prenant des initiatives tactiques en fonction du contexte. «Je vous donnerai, au reste, selon que les évènements l'exigeraient, toutes les instructions qui vous seront nécessaires, ne doutant pas que, dans toutes les circonstances, vous n'agissiez conformément à l'esprit de la guerre que nous faisons. » (Napoléon au général Joubert le 15 mars 1797).

Napoléon, module son management en fonction de l'urgence et de la proximité au terrain. « Vous devez sentir Général qu'à la distance où vous êtes, et avec votre grade, ce n'est pas un ordre littéral qui doit vous faire mouvoir mais la masse des évènements. » (Napoléon à Marmont le 19 juin 1809).

Face à l'état d'urgence immédiate, le management sera davantage autoritaire comme dans la communication descendante et l'élaboration des objectifs et des ordres stratégiques. Il faut agir vite sous la pression.

Par contre dans un état d'urgence « différée » où la pression est moins forte à court terme, le style de management devient davantage paternaliste et persuasif. Napoléon est plus dans l'anticipation notamment pour des facteurs liés à la motivation et l'intérêt porté aux subordonnés.

De plus, le critère de la proximité au terrain dans un contexte d'urgence forte nécessite de tendre vers un management plus consultatif dans certains cas comme lorsque Napoléon encourage les chefs de corps d'armée à prendre des initiatives de manière contingente.

Après avoir vu les particularités du leadership de Napoléon, lesquelles sont un des fondements majeurs de sa pensée managériale, nous allons maintenant mettre l'accent sur un $2^{\text {ème }}$ aspect primordial de son style de commandement et de management des hommes et des ressources. Napoléon est en effet un scientifique qui excelle dans les mathématiques (artilleur et membre de l'Institut de France à la section des arts mécaniques de la section des sciences dès 1797). On le voit à travers certains de ses principes de vitesse et de mobilisation des forces illustrés par ses propres réflexions militaires : «La force d'une armée, comme la quantité de mouvement en mécanique, s'évalue par la masse multipliée par la vitesse »(COLSON, op.cit..). Ces principes militaires constituent l'un des axiomes de sa pensée managériale pour gérer l'urgence. 


\subsection{Principes (militaires) de rapidité et de mobilité}

Napoléon s'est ici inspiré des écrits de Guibert, De Bourcet ou du Teil. Héritier intellectuel de ces penseurs, il a su utiliser certains de leurs principes de manière contingente à son environnement et en faire une synthèse d'ensemble qui se traduira notamment dans la Grande Armée. Le système divisionnaire de Guibert en est une des illustrations. Plus qu'un théoricien, Napoléon est avant tout un stratège. Confronté à des urgences de natures diverses (militaires, politiques, économiques), Napoléon doit agir vite et fort (SUCHET, op.cit.). Imposer la confrontation est possible grâce à la puissance flexible de la division et du corps d'armée. Il doit vaincre et rapidement. Il y a donc urgence dans la durée. Mais à cette pression constante s'ajoute celle du moment de la bataille que l'on peut qualifier d'instantanéité de l'urgence.

L'Empereur est un stratège, Il applique ses axiomes stratégiques systématiquement (concentration des forces, vitesse, flexibilité) afin d'anticiper l'urgence. Pour appliquer ces principes il prend en compte le contexte momentané de l'urgence et s'appuie au niveau tactique et opérationnel sur des officiers rompus au combat et expérimentés auxquels il délègue des pouvoirs importants (exemple de Murat). Il y a donc urgence dans la durée et dans l'instantanéité du moment.

L'objectif de Napoléon est de se retrouver plus puissant et nombreux que l'adversaire en un point précis pour « le battre en détails » (alors que confronté à de nombreuses coalitions il est arithmétiquement presque toujours en très nette infériorité). Les divisions par leur mobilité et leur autonomie permettent de couvrir un large terrain, jetant ainsi le trouble chez l'ennemi sur les points d'attaque. Qualités qui leur permettront par la suite de converger rapidement et en nombre sur l'un des points faibles de l'ennemi.

Pour cela, son management s'appuie sur deux éléments récurrents dans la construction progressive des campagnes qu'il mène :

- la mobilité des troupes,

- la vitesse de déplacement des divisions qui permet justement la réalisation du point précédent. « Activité, activité, vitesse » (Napoléon à Masséna, 1809).

Napoléon, dès sa jeunesse va étudier les campagnes et concepts appliqués par les penseurs militaires que sont Frédéric II, Guibert (1775), le Maréchal De Broglie et De Bourcet (1775).

Lors de la bataille, il y a deux ordres possibles qui assurent la prédominance d'une arme. 
- L'ordre mince appelé aussi linéaire qui donne à l'infanterie un rôle capital. Cette dernière répartit en plusieurs rangs s'étire tout au long du front de la bataille.

- L'ordre profond, où les troupes progressent en colonnes étroites et qui sert en particulier pour les déplacements. Dans le combat, elle met en valeur la cavalerie dont on attend la puissance de choc d'une charge en colonne.

Avec l'apparition des armes à feu puis le remplacement progressif du mousquet par le fusil -plus commode à recharger- la puissance de feu de l'infanterie lui donne un avantage décisif. C'est donc l'ordre mince qui est préconisé. Ainsi au XVIIIème siècle, l'infanterie de l'armée française s'aligne sur trois rangs resserrés séparés par environ trois cent pas de deux à trois autres rangs (en réserve). La cavalerie est le plus souvent divisée en deux et positionnée sur chaque flanc. Dans cette configuration, l'artillerie placée devant l'infanterie joue surtout un rôle en début de combat, son manque de mobilité l'empêchant de peser sur la suite des évènements. Dans un système privilégiant l'ordre mince au combat, il est très difficile de forcer un ennemi à combattre s'il ne le souhaite pas. En effet, le déplacement des troupes ajouté à l'espacement en ligne sur le champ de bataille est très chronophage et peut laisser le temps à l'adversaire de se retirer. Le temps de se remettre en colonne pour le poursuivre et il est déjà loin !

GUIBERT (op.cit.) va alors préconiser en 1770, l'ordre mixte, compromis entre ordre mince et profond pour passer de l'un à l'autre afin de bénéficier des avantages des deux systèmes. Cela n'est possible que si l'on réorganise la structure de l'armée en unités mobiles et autonomes comme le bataillon (environ 800 hommes). C'est la naissance de la division dont une des unités de base sera le bataillon composé au niveau de l'infanterie de 4 compagnies de 200 hommes). Les divisions de l'infanterie seront ainsi regroupées dans le corps d'armée en synergie avec les divisions de cavalerie ainsi que la division d'artillerie laquelle en bénéficiant du système de Gribeauval est rendue beaucoup plus légère donc plus mobile et efficace sur la durée du combat.

Avec cette structure "divisionnelle » beaucoup plus mobile et agile, Napoléon peut forcer l'ennemi à combattre sur le champ de bataille qu'il a la plupart du temps choisi. Il répond ainsi à l'urgence de sa situation.

Napoléon s'appuie aussi sur la formation en carré. Les côtés des carrés sont formés par trois rangs de soldats (deux à la fin de l'Empire). C'est à partir de la colonne (ordre profond) qu'il est le plus facile et rapide à mettre en place («Formez le carré »). Le bataillon (vu précédemment) est la plus petite unité permettant de former un carré pérenne soit environ 800 
hommes avec la réserve et les drapeaux au centre et l'artillerie aux coins (endroit le plus fragile). Pour être efficace le tir ne doit pas être déclenché trop tôt ce qui exige un grand sans froid et beaucoup d'expérience de la part des troupes et de leur commandement. Une fois le danger passé le carré se reforme en colonne («Formez les divisions »). La force du carré tient dans sa cohésion qui lui donne une puissance de feu majeure.

Les moyens pour y arriver sont d'ordre managériaux notamment sur le plan organisationnel. Dans ses «entreprises » que sont les campagnes, la pensée managériale de Napoléon a donc construit, à travers la Grande Armée, un outil efficace que l'on peut rétrospectivement analyser en mobilisant des modèles théorisés et appliqués par la suite. Napoléon, par sa pensée managériale et sa mise en action est peut-être un précurseur méconnu.

\section{DES MOYENS D'ACTION ORIENTES VERS LA GESTION DE L'URGENCE}

Napoléon va se servir de façon combinée de la communication et de l'organisation. Ces deux outils permettant la cohésion et la concentration de ses forces, éléments fondamentaux recherchés dans le management de l'urgence développé par Napoléon.

\subsection{La communication}

\subsubsection{La communication institutionnelle}

Celle-ci lui permet d'assoir sa légitimité et va susciter l'adhésion de ses troupes, atout primordial en situation d'urgence où la cohésion est essentielle.

Tout d'abord, examinons l'aspect symbolique, sur le plan de la communication visuelle, du choix des emblèmes impériaux, l'Aigle et l'Abeille. Au conseil d'Etat, le 12 juin 1804 (23 prairial an XII) se pose la question de l'emblème du pouvoir impérial. On hésite entre le coq, l'éléphant et le lion. Les conseillers choisissent le coq choix réfuté par l'Empereur qui préfère dans un premier temps le lion puis dans un deuxième temps l'aigle. Ce choix est à rapprocher en tant que signe distinctif de ce que peut être le logo d'une organisation, support d'un message et repère dans le temps.

En choisissant l'Aigle, Napoléon fait référence aux légions romaines (1'Antiquité est alors à la mode), mais un aigle stylisé Carolingien ce qui renvoie aussi à Charlemagne. Romains et Carolingiens sont tous deux fondateurs d'empires dont les prestiges sont séculaires. Napoléon a besoin de cette dialectique pour assoir le sien face aux monarchies européennes beaucoup plus anciennes. Dans la même veine, les abeilles sont le symbole de l'immortalité et de la résurrection, cette symbolique permettant de rattacher la nouvelle dynastie aux origines de la France. En effet, des abeilles auraient été découvertes dans le tombeau de Childéric Ier, fondateur en 457 de la dynastie mérovingienne et père de Clovis. Elles sont considérées comme 
le plus ancien emblème des souverains de la France. On peut aussi voir dans l'Abeille l'aspect industrieux et ordonné de cet animal qui construit la ruche productrice (la France) sous la direction de sa Reine (son Empereur).

Ensuite, deuxième facette de cette communication institutionnelle, le poids des images. Napoléon s'en est beaucoup servi dans sa propagande enjolivant la réalité comme par exemple lors du passage du pont d'Arcole. Les cérémonies de remise de Légions d'Honneur participent également à la diffusion de l'image ainsi que les tableaux commandés par Napoléon Bonaparte au peintre David («Bonaparte franchissant le Grand-Saint-Bernard», «le sacre de Napoléon »). Ces œuvres auront vocation à servir la propagande Napoléonienne à moyen ou long terme. Par contre, les réalisations d'Isabey, portraitiste et miniaturiste, plus aisément reproductibles seront davantage mobilisables sur le court terme (BOUDON, 2000).

\subsubsection{La communication interne}

En interne, Napoléon utilise la communication descendante, à la fois de manière formelle mais aussi informelle.

Formellement, les proclamations aux soldats en sont un des exemples les plus aboutis. Ces textes lus avant la bataille ou à l'occasion d'un évènement important utilisent à bon escient une sémantique évocatrice. L'accent est mis -si l'on se réfère à JAKOBSON (1960)- sur les fonctions :

- interpellatives « Soldats, vous avez en quinze jours remporté la victoire, pris 21 drapeaux, 55 pièces de canon, plusieurs places fortes, conquis la partie la plus riche du Piémont; vous avez fait 15000 prisonniers, tué ou blessé près de 10000 hommes ». «Vous vous étiez jusqu'ici battus pour des rochers stériles. Dénués de tout vous avez supplée à tout. Vous avez gagné des batailles sans canons, passé des rivières sans pont, fait des marches forcées sans souliers, bivouaqué sans eau-de-vie et souvent sans pain. » (Proclamation à l'armée d'Italie le 26 avril 1796).

- émotives et interpellatives «Soldats ! Je suis content de vous. Vous avez à la journée d'Austerlitz, justifié tout ce que j'attendais de votre intrépidité ; vous avez décoré vos aigles d'une immortelle gloire. » (Proclamation après Austerlitz du 3 décembre 1805).

- poétiques «Les phalanges républicaines, les soldats de la liberté étaient seuls capables de souffrir ce que vous avez souffert. » (Proclamation à l'armée d'Italie le 26 avril 1796). « Allez, et pensez que du haut de ces monuments, quarante siècles vous observent » (Campagne d'Egypte, 1798). 
Ces proclamations que l'on retrouve dans les campagnes de Napoléon sont restées célèbres. Elles interviennent à des moments clés où il y a urgence à mobiliser les soldats en termes de cohésion.

Toujours dans la communication formelle, il faut souligner le rôle prépondérant de l'Etat-Major et de son chef jusqu'en 1814, le Maréchal Berthier. Ce dernier assiste jour et nuit Napoléon Bonaparte et doit être prêt à saisir la volonté de son chef ce qui nécessite une grande capacité d'écoute et de résistance physique. Ensuite en tant qu'émetteur, Berthier et son EtatMajor doivent transmettre les ordres clairement, sûrement et rapidement tout le long de la ligne hiérarchique. Exercice hautement difficile car les lignes de transmission sont très étirées par l'étendue des champs de bataille. Consciencieux, Berthier n'hésite pas à envoyer plusieurs officiers ou soldats porter le même ordre car le taux de réussite de la transmission est très aléatoire. L'Etat-Major collecte les renseignements et surveille le ravitaillement et la bonne exécution des décisions, ainsi que le travail des services annexes comme par exemple les relevés qu'exécutent les ingénieurs-géographes dans les pays occupés, pour la logistique et la progression de l'armée.

De manière informelle, les exemples sont nombreux où l'Empereur communique de façon libre et spontanée avec ses soldats, par exemple la veille de la bataille d'Austerlitz. L'Empereur connait même le nom de nombreux soldats vétérans. Cela crée de la proximité. De plus en campagne, Napoléon vit comme ses soldats sans apparat (la fameuse redingote grise) en partageant un mode de vie frugal, en synchronisation avec leur quotidien. Cette manière de fonctionner vise à renforcer la cohésion propice à faire face à l'urgence.

\subsection{L'organisation}

Les moyens d'action de Napoléon reposent sur des principes organisationnels qui doivent renforcer la réactivité et la mobilité de l'arsenal militaire napoléonien. Il lui faut :

- disposer d'une structure rationnelle et efficiente pouvant faire face à la complexité de l'environnement,

- assurer la cohésion des troupes dans l'urgence en s'appuyant sur la capitalisation de l'expérience de certaines unités,

- concilier rationalité et pragmatisme dans la prise de décision.

Nous pouvons, sans prétendre à l'exhaustivité, illustrer ces principes, précédemment évoqués, à travers l'organisation de la Grande Armée et analyser des exemples d'application lors des campagnes militaires. 


\subsubsection{L'organisation de la Grande Armée : une structure staff and line}

La grande armée est sous le commandement direct de l'Empereur et elle est organisée sur la base d'une structure staff and line pour faciliter la réactivité des troupes et leur mobilité.

\section{Le staff}

Napoléon est directement le chef suprême de l'armée. C'est sur le Grand Quartier Général (GQG) qu'il s'appuie. Ce dernier est composé de deux entités la Maison de l'Empereur et l'Etat-Major.

En outre, chaque Maréchal à la tête d'un corps d'armée, ainsi que les généraux de division, ont leur propre Etat-Major. C'est sur les officiers de liaison qui composent l'Etat-Major (aides de camp) que reposent la communication entre le commandement, les troupes sur le terrain et une partie de l'organisation des services logistiques (distribution de nourriture et transport du matériel).

\section{La " Task force " : la Garde et la réserve de cavalerie}

Napoléon crée et organise « la réserve ». Il s'agit dans la stratégie de Napoléon de garder des troupes en réserve sous son commandement direct pour, au besoin, favoriser l'application de son principe le plus absolue : être plus nombreux que l'ennemi pour le battre en détails lors d'une bataille décisive. Cette réserve (la garde) sera constituée notamment par la vieille, la moyenne et la jeune garde ainsi que par la réserve de cavalerie. Troupes d'élite, ces ressources sont rares, stratégiques et lui donnent un avantage décisif notamment en situation d'urgence. L'intervention de cette task force doit s'avérer décisive au bon moment et constitue ce qu'il appelle «l'évènement ». Ces réserves comme les corps d'armés doivent avoir des qualités :

- de mobilité

- de résilience

- de puissance

Pour être efficace dans l'urgence et surprendre l'ennemi soit par une «manœuvre sur les arrières » (comme à Iéna en 1806 par exemple) soit par une «manœuvre centrale » pour diviser l'ennemi et l'empêcher en urgence de se regrouper » (cas de la campagne de France de 1814).

Tout au long de l'épopée Napoléonienne, l'utilisation de la Garde est corrélée avec la montée de l'urgence comme l'illustre le tableau 2. Selon que l'urgence est jugée de faible à extrêmement forte, Napoléon mobilise tout ou partie de la Garde (tableau 2). 
Tableau 2. La mesure de l'urgence à travers la corrélation avec l'utilisation de la garde dans les campagnes de 1805 à 1815

\begin{tabular}{|c|c|c|c|c|}
\hline Dates & Campagnes & Batailles & Utilisation de la Garde & Urgence \\
\hline 1805 & Autriche & Ulm & Contre la cavalerie de la garde Russe & Faible \\
\hline 1806 & Prusse & Iéna & Présente mais n'intervient pas & Très faible \\
\hline \multirow[t]{2}{*}{1807} & \multirow[t]{2}{*}{ Pologne } & Eylau & Cavalerie (charge de Murat) & Forte \\
\hline & & Friedland & Infanterie et quelques unités de cavalerie & Moyenne \\
\hline \multirow[t]{4}{*}{1808} & \multirow[t]{4}{*}{ Espagne } & Madrid & Charges urbaines & Forte \\
\hline & & Medina del rio seco & Dragons et fusillers-chasseurs & Forte \\
\hline & & Benavente & Chasseurs à cheval & Forte \\
\hline & & Charge de Somossierra & $\begin{array}{c}\text { Chevau-légers Polonais (entrent après dans la } \\
\text { Garde) }\end{array}$ & Très forte \\
\hline \multirow[t]{2}{*}{1809} & \multirow[t]{2}{*}{ Autriche } & Aspern et Essling & Tirailleurs-grenadiers/Vieille Garde & Forte \\
\hline & & Wagram & Cavalerie de la Garde & Forte \\
\hline $\begin{array}{c}1810- \\
1811\end{array}$ & \multirow[t]{2}{*}{ Espagne } & $\begin{array}{c}\text { Salinas, Viana, Arlavan, } \\
\text { Acedo et Oyon }\end{array}$ & \multirow{2}{*}{ Jeune Garde avec voltigeurs et tirailleurs } & Forte \\
\hline 1812 & & $\begin{array}{l}\text { Luzzara, Acedo, } \\
\text { Arranda, Valmaseda, }\end{array}$ & & Forte \\
\hline \multirow[t]{2}{*}{1812} & \multirow[t]{2}{*}{ Russie } & La Moskowa & Artillerie & Forte \\
\hline & & $\begin{array}{l}\text { Krasnoie, bérézina, } \\
\text { Kowno. }\end{array}$ & Toute la Garde & $\begin{array}{l}\text { Extrêmement } \\
\text { forte }\end{array}$ \\
\hline \multirow[t]{3}{*}{1813} & \multirow[t]{3}{*}{ Allemagne } & Lutzen & Jeune Garde & Forte \\
\hline & & Dresde & Jeune Garde & Forte \\
\hline & & Leipzig & La Garde & Très forte \\
\hline 1814 & France & $\begin{array}{c}\text { Bar-sur-Aube, Brienne, } \\
\text { La Rothière, Château- }\end{array}$ & La Garde & Très forte \\
\hline 1815 & Belgique & Ligny, Waterloo & La Garde & $\begin{array}{l}\text { Extrêmement } \\
\text { forte }\end{array}$ \\
\hline
\end{tabular}

Napoléon -contrairement aux Russes, Autrichiens et Prussiens- concentre dans la réserve de cavalerie la quasi-totalité de la cavalerie lourde des corps d'armée les jours de combat. Placée sous les ordres de Murat, elle lui permet d'avoir une grande puissance de choc lorsqu'elle intervient en urgence.

La cavalerie lourde combat en général avec 25 hommes de front ce qui fait un quadrilatère d'environ $50 \mathrm{~m}$ sur 6 (BRUN 2008). Les cavaliers partent au trot puis à 200 pas au galop et enfin à 80 pas au galop de charge. Mais c'est surtout la compacité qui compte par rapport à la vitesse. L'impact est aussi psychologique par l'impression de puissance que donne la cohésion. L'exemple de la bataille d'Eylau illustre l'efficacité de la réserve de cavalerie face à l'urgence imminente. Davout malgré une grande efficacité est en difficulté. Pour le renforcer Napoléon envoie Augereau, mais ses troupes sont victimes de la tempête de neige qui les aveugle. Devant l'urgence, Napoléon fait alors donner la Garde puis la réserve de cavalerie soit les 58 escadrons de Murat et les 16 de la Garde de Bessières. En tout près de 18000 cavaliers. 


\section{Le line}

Le pivot de l'organisation générale est le corps d'armée à la tête duquel est placé par 1'Empereur, un Maréchal d'Empire.

Il existera jusqu'à 14 corps d'armée. Les corps d'armées sont des « divisions » (au sens de Chandler, op.cit.) et à l'intérieur de chacune on retrouve de manière fonctionnelle les 3 grandes composantes que sont l'infanterie, la cavalerie et l'artillerie sous la forme de divisions (au sens militaire) en nombres variables avec à la tête de chacune un Général de division

L'organisation est pyramidale avec les mêmes éléments de niveau à savoir la brigade, le régiment, le bataillon puis la compagnie (approche quasi-fractale). Classique du domaine militaire très hiérarchisé, ce dernier point met en lumière le rôle clé d'une ligne hiérarchique très allongée au sens de MINTZBERG (1984) faisant le lien entre le sommet stratégique (le Grand Quartier Général) et le centre opérationnel que sont les troupes sur le terrain. Maréchal, Général de Division, Général de Brigade, Colonel, Capitaine, Lieutenant, Sergent, Caporal composent cette ligne hiérarchique qui est à la fois un handicap mais aussi un atout. Handicap par le risque de lourdeur et de perte d'information tout au long du processus de communication qu'il soit ascendant ou descendant. La ligne hiérarchique est cependant également un atout par la présence des sous-officiers qui assurent non seulement l'enseignement militaire, mais aussi la transmission des valeurs voire l'éducation des conscrits (exemple du camp de Boulogne). Dans cette organisation, le rôle de l'Etat-Major, et du Maréchal Berthier qui le dirige de 1805 à 1814, est primordial par la clarté et la précision de ses ordres. Il semble d'ailleurs que l'absence de Berthier en 1815 à Waterloo ait pesée lourd dans le résultat de la bataille (WIEVIORKA et al., 2018).

Il est clair que la Grande Armée construit son efficacité en amalgamant de manière efficiente staff and line, créant ainsi des synergies, pour la rendre réactive et mobile. Les activités opérationnelles (la logistique amont, le déploiement des troupes, la logistique aval d'occupation du territoire conquis) sont distinguées des activités de soutien (le génie, les pontonniers, le service de santé - avec des chirurgiens de talents comme Larrey, Desgenettes, Percy, Parmentier- les services de renseignement, la cartographie, la gendarmerie). Mais ces deux formes d'activité sont aussi reliées en permanence par la structure staff and line afin de renforcer la rapidité d'exécution des opérations militaires.

\subsubsection{La structure de la grande armée : flexibilité et agilité pour faire face à l'urgence}

Certains éléments de l'organisation précédemment décrites sont de nature administrative. Ils ne sont que les pivots nécessaires à l'application efficace des axiomes 
napoléoniens que sont la vitesse et la mobilité pour assurer en terme de résultat la concentration des troupes. L'idée est de constituer des ensembles assez forts pour résister à l'ennemi et en même temps assez proches pour se soutenir et se rassembler et ainsi battre l'ennemi par le nombre (TRANIE, 1999). On peut citer comme exemple, le cas de la division Friant à Austerlitz en 1805 qui, en résistant suffisamment de temps, permet la concentration des forces françaises sur le plateau de Pratzen et offre à Napoléon l'opportunité de «tourner » son ennemi et de le prendre à revers.

Ces savantes manœuvres ne sont possibles qu'à trois conditions :

- les renseignements portés à la connaissance de l'Empereur doivent être exacts ce qui est la plupart du temps le cas. Une exception notable cependant avec la bataille de Marengo (San Giuliano) en 1800 où, trompé par des informations inexactes, Bonaparte éloigne la division Desaix. En infériorité numérique, il manque de peu d'être écrasé avant un prompt retour de Desaix due à la dextérité de ses troupes.

- ce qui nous amène à la condition de la mobilité. Elle est extrême pour les troupes françaises qui disposent dans leurs rangs des meilleurs marcheurs. A titre de comparaison, quand le soldat français fait en moyenne $4 \mathrm{~km} / \mathrm{h}$, ses homologues anglais et prussiens n'en réalisent que 3 et seulement 2 pour les autrichiens et les russes. Une troupe française peut donc sur une journée de 10 heures de marche abattre $40 \mathrm{~km}$ voire plus, comme les troupes de Davout qui -pour soutenir dans l'urgence la division Friant précédemment cité- vont réaliser en 48 heures, lors de la bataille d'Austerlitz, $110 \mathrm{~km}$ pour être sur le théâtre des opérations à temps. Ainsi grâce à cette vitesse, Napoléon a pu tromper ses ennemis en leur faisant croire qu'il n'état potentiellement pas en force sur le terrain. L'armée française présente donc un avantage concurrentiel majeur sur ses adversaires qui sera par la suite maintenu, malgré l'inexpérience des nouveaux soldats, par une réorganisation des colonnes de marche. Par exemple lors de la bataille de Rivoli (14 janvier 1797) pendant la $1^{\text {ère }}$ campagne d'Italie, une $4^{\text {ème }}$ armée autrichienne a été constituée pour venir en aide à Mantoue assiégée. Il est urgent de faire face à la diversion des Autrichiens sur Joubert qui occupe le plateau de Rivoli. Il s'agira ensuite de couper la route de Vérone qui dessert Mantoue. La célérité des troupes de Masséna venues renforcées Joubert va sceller le sort de la bataille. Les Autrichiens ont été trompés par la vitesse de l'armée de Bonaparte et se croyant à tort potentiellement en supériorité numérique ont trop étendu leurs lignes d'encerclement du plateau (TRANIE, 1999).

- la structure doit être souple, modulable et adaptable de manière contingente à un environnement et un contexte évolutifs. Ainsi les divisions sont enlevées, regroupées ou augmentées selon les corps d'armée en fonction des besoins. MIQUEL (2005) nous 
explique ainsi en détails ces restructurations stratégiques. Ainsi, par exemple, le $1^{\text {er }}$ décembre (veille de la bataille d'Austerlitz), Napoléon enlève au $1^{\text {er }}$ corps d'armée du Maréchal Bernadotte une division de cavalerie légère, commandée par Kellermann, pour les attribuer au corps d'armée du Maréchal Murat, dont la charge s'avèrera décisive. De même, le $27^{\text {z̀me }}$ de cavalerie légère du Général Frère quitte le $1^{\mathrm{er}}$ corps pour renforcer la Division Drouet très exposée (ibid.). C'est bien l'Empereur qui décide de tout et la réaction de dépit de Bernadotte montre qu'il ne connait pas le plan de Napoléon expliquant de manière logique ces redéploiements.

L'exemple de la bataille de Wagram (5-6 juillet 1809) l'illustre. Réfugié sur l'île Lobau sur le Danube, Napoléon en fait sa base logistique. Les Français traversent le Danube le 5 juillet pour se retrouver sur la plaine de Wagram. Il compte enclencher une attaque de diversion pour tourner les Autrichiens qui eux se sont réfugiés derrière un affluent du Danube. Le 6 juillet à 4h, les Autrichiens lancent une attaque générale de front maitrisée au niveau de l'aile droite de l'armée française (Davout), mais qui ébranle le centre (Bernadotte) et surtout exerce une dangereuse pression sur l'aile gauche française formée de la division Boudet qui se bat à un contre cinq. Ce dernier se replie sur Essling et les Autrichiens se rabattent sur le Danube pour couper les Français de leurs arrières. Dans l'urgence, Napoléon demande à Masséna d'arrêter la progression de l'ennemie en étant protégé par sa réserve de cavalerie (cuirassiers de Saint Sulpice). Pour réaliser cela, Masséna doit exécuter une périlleuse marche de flanc -s'exposant aux divisions de l'ennemi- qu'il réussit grâce à la flexibilité de la structure des troupes et à son habileté. Le déplacement des troupes de Masséna crée un vide qui doit être comblé par Mac Donald qui reçoit l'appui de Wrède et de la Jeune Garde dans des flux physique et d'information combinés. La réussite de la manœuvre de Masséna passera par l'optimisation des 3 armes au sein de la division en mouvement. La mobilité de l'artillerie déblayant le chemin, tandis que l'infanterie tantôt en progression en colonne par régiments en colonne puis formant le carré en bataillon reçoit l'appui de la cavalerie Lassalle. Ayant stabilisé en urgence son flanc gauche qui fixe une partie des forces Autrichiennes, Napoléon va pouvoir enclencher en début d'aprèsmidi son offensive victorieuse au centre. En choisissant, un de ses Maréchaux les plus intrépides, Napoléon a confirmé qu'un des modes de management de l'urgence est de laisser à des subordonnés compétents et expérimentés la liberté d'agir et de prendre des initiatives dans l'exécution de la stratégie d'un point de vue tactique et opérationnel (CANETTI et al., 2013). Si l'on se réfère à RIVELINE (op.cit., p.90) pour faire face à l'urgence, il faut une « réponse programmée » comme l'anticipation de Napoléon. Mais souvent c’est « la débrouillardise » qui prévaut et peut marcher s'il y a habileté et dévouement des opérateurs (militaires expérimentés 
et dévouement à Napoléon comme l'exemple de Masséna le démontre) avec augmentation des zones d'incertitudes que seul un habitué de ce type de contexte peut optimiser.

Dans tous les cas, l'ensemble de ces éléments d'organisation associés à des principes militaires forts ont pour but et résultat de construire une cohésion d'ensemble des hommes comme de l'organisation.

\section{LES RESUltats DE SA PENSEe AXIOMATIQUE ET DE SES MOYENS D'ACTION}

\subsection{La cohésion}

La cohésion est le premier et le principal résultat repérable issu de la pensée managériale napoléonienne et de sa mise en action. La cohésion est la propriété d'un ensemble dont toutes les parties sont solidaires. C'est une force qui permet l'adhésion et qui dépend de l'attractivité du groupe. Cette cohésion est ainsi le résultat de divers éléments énumérés précédemment appartenant à la pensée managériale (leadership) ou à ses modes d'action (communication et organisation).

Tout d'abord, comme vu plus haut, le leadership napoléonien se construit autour de valeurs partagées. Le soldat de la période napoléonienne attache une grande importance à l'égalité et à la reconnaissance des mérites (ascenseur social). Napoléon sait répondre à ces attentes. «Il y a un bâton de maréchal dans chaque giberne de soldat » aurait dit Napoléon, idée fortement persistante sous l'Empire (TULARD, 2012). Les exemples de Masséna, Victor, Bessières ou Murat semblent le démontrer. Ces valeurs sont également transmises par des signes de reconnaissance (par exemple la Légion d'Honneur que Napoléon crée).

Par sa proximité au soldat, puisqu'il est sur le terrain et le champ de bataille, Napoléon partage aussi le quotidien du soldat ce qui pousse l'adhésion à sa personne et à ses principes. Ceci renvoie à ses modes de communication, déjà illustrés, qui créent de la cohésion parmi ses troupes. Ainsi les objectifs militaires sont en général clairement communiqués et lisibles (ex campagnes d'Italie, Austerlitz), même s'ils le sont moins vers la fin de l'épopée à l'exception de la campagne de France. Napoléon sait écouter voire déléguer. Surtout il stratifie à bon escient les responsabilités de chacun (Etat-major, Maréchaux, généraux de Division de brigade etc.). On oublie de surcroit trop souvent le rôle des bas officiers dans la communication des valeurs napoléoniennes (à rapprocher du management intermédiaire aujourd'hui remis en cause dans les entreprises). Dans l'urgence, ces derniers sont la meilleure courroie de transmission.

Cette cohésion est aussi le résultat de l'organisation militaire napoléonienne illustrée plus haut. Etant bien formés quant aux techniques de marche, le déplacement des troupes se fait donc de manière rapide et efficace dans un mouvement d'ensemble, de manière coordonnée. 
Lors de la bataille d'Austerlitz les déplacements de troupes -parfois éloignées quelques jours avant la bataille- s'effectuent dans une très grande coordination due à une grande homogénéité du commandement (MIQUEL, 2005). Le partage de connaissances entre les officiers du terrain, durant les années de campagne en commun, a créé un effet d'expérience propice à cela. La Grande Armée est capable de résister à un adversaire plus puissant qu'elle, car dans l'urgence, elle peut structurer, par expérience, ses forces « divisionnelles », de manière centripète, pour assurer une cohésion forte et battre l'ennemi sur le terrain. La cohésion est également développée par les valeurs véhiculées par l'armée de Napoléon comme la reconnaissance du talent, le mérite, l'opiniâtreté, l'acceptation des objectifs, les valeurs républicaines mais aussi l'admiration face à leur leader charismatique qui n'hésite pas à donner l'exemple en s'exposant au danger.

\subsection{Rationalité et pragmatisme pour concentrer ses forces}

L'Empereur est un scientifique qui aime la précision de la rationalité et ne laisse rien au hasard. Il est dans l'anticipation ce qui lui permet de faire face à des situations d'urgence. Sa connaissance des moyens dont il dispose est très étendue. Son diagnostic stratégique est précis tant en interne, en termes de forces et faiblesses, qu'en externe, en termes de menaces et d'opportunités.

Nous avons déjà vu comment Napoléon tire parti des forces de son armée en termes de vitesse de déplacement et d'organisation flexible. La Grande Armée est l'instrument qui permet à Napoléon d'assurer la réunion de ses forces dans sa seule main et sous son unique commandement en cumulant «les avantages de la dispersion et de la concentration» (COLSON, 2011). Ainsi, les forces doivent être réunies -de manière éloignée mais suffisamment proches pour se porter secours- la veille d'une action, sans pouvoir être battue séparément par l'ennemi. A sa proximité elles sont réunies pour être concentrée sur une partie de l'armée ennemi et la battre par supériorité numérique (COLIN, 1900). Cela peut passer par des marches forcées très importantes comme celles du corps de Bernadotte à Wagram en 1809 qui parcourent dans les jours précédents la bataille près de $150 \mathrm{kms}$ pour se rapprocher du lieu de l'affrontement! C'est dire l'importance accordée à ce principe par 1'Empereur. «Un bataillon quelques fois décide d'une journée » (Napoléon cité par Montholon dans ses mémoires). La mobilité et l'organisation flexible permettent de surcroit d'étirer le front (donc l'ennemi) pour ensuite le prendre de vitesse par la concentration. A titre d'exemple, lors des campagnes de 1805 et 1806, la ligne de front couvre un espace d'une centaine de kilomètres puis se rétrécit la veille des batailles à 40 ou 30 kilomètres. Ce qui fait dire à Alexandre 
s'adressant au Général Savary après la bataille d'Austerlitz « Vous étiez moins nombreux que moi et cependant, vous étiez plus forts partout ». Par analogie, on peut y voir un filet à plus ou moins larges mailles qui se déploie et se resserre sur l'ennemi.

Napoléon est bien conscient -toujours sur le plan interne- que l'une des faiblesses de la cavalerie française se situe dans le manque de chevaux et la possibilité de les entretenir. Pour pallier à des situations d'urgence futures, il anticipe cela par exemple lors de la préparation de l'invasion de l'Angleterre, à partir de 1804. Les cavaliers embarqués ne disposent pas tous de chevaux et de vivres qu'ils pourront obtenir en Angleterre (principe aussi appliqué pour les troupes).

Mais face à l'urgence, Napoléon ne fait pas qu'anticiper, il saisit aussi les opportunités offertes sur le théâtre des opérations. Sa connaissance du terrain grâce à sa prédilection pour la géographie lui permet d'utiliser voire de choisir à son avantage le champ de bataille comme par exemple lors des campagnes d'Italie (Rivoli) ou à Austerlitz (sur le Plateau de Pratzen et dans l'étang de Kobelnitz) bien aidé par le géographe Bacler d'Albe (PIGEARD, 2004). Quant aux menaces venant des troupes coalisées adverses, Napoléon les prend en compte en appliquant son principe déjà évoqué de les diviser pour «mieux les battre en détail » (L'Empereur au Maréchal Lannes, 1806).

La rationalité de Napoléon est-elle limitée en termes de capacité cognitive et d'information disponible (au sens de SIMON - 1957) ? Sur le premier plan, l'Empereur a une très grande confiance dans ses capacités. Il déploie en ce sens un optimisme inébranlable même après certains échecs comme lors de la campagne de Russie ou au cours de la campagne de France. Cependant, sur le plan de l'information, Napoléon a bien conscience qu'il s'agit d'un domaine perfectible, ce qui l'a amené tant sur le plan extérieur qu'intérieur à développer des organes de renseignement performants parfois parallèles et secrets.

De même, l'urgence crée des zones d'incertitude (CROZIER et FRIEDBERG, op.cit.), ce qui donne des espaces de liberté sur le terrain dans lesquels Napoléon peut s'engouffrer puisque par son anticipation (figure 2), il est justement en situation d'asymétrie d'information favorable face à ses adversaires. Ici encore la cavalerie -légère cette fois- joue un grand rôle. Elle assure deux missions à ce niveau (BRUN, 2008) :

- La sureté : la cavalerie patrouille devant les troupes et masque ainsi à l'ennemi les forces et mouvements de l'armée française (asymétrie d'information). Ainsi, en 1805, avant 
Austerlitz c'est un réseau de $200 \mathrm{kms}$ de patrouilles montées qui est mis en place du Danube aux montagnes de Bohême.

- La recherche de l'information et du renseignement: il s'agit pour une brigade de cavalerie légère de 15 à 25 hommes, d'explorer dans toutes les directions (jusqu'à 30 $\mathrm{kms}$ ). Parfois cette recherche est plus massive et anticipatrice comme celle de Murat en 1806 avant Iéna.

Ainsi, d'une manière générale, les aléas de terrain créent des zones d'incertitudes auxquelles la Grande Armée fait face grâce aux moyens d'action de la pensée managériale napoléonienne précédemment évoqués (figure 2).

Figure 2. Urgence et zones d'incertitude dans le management napoléonien (source : auteurs)

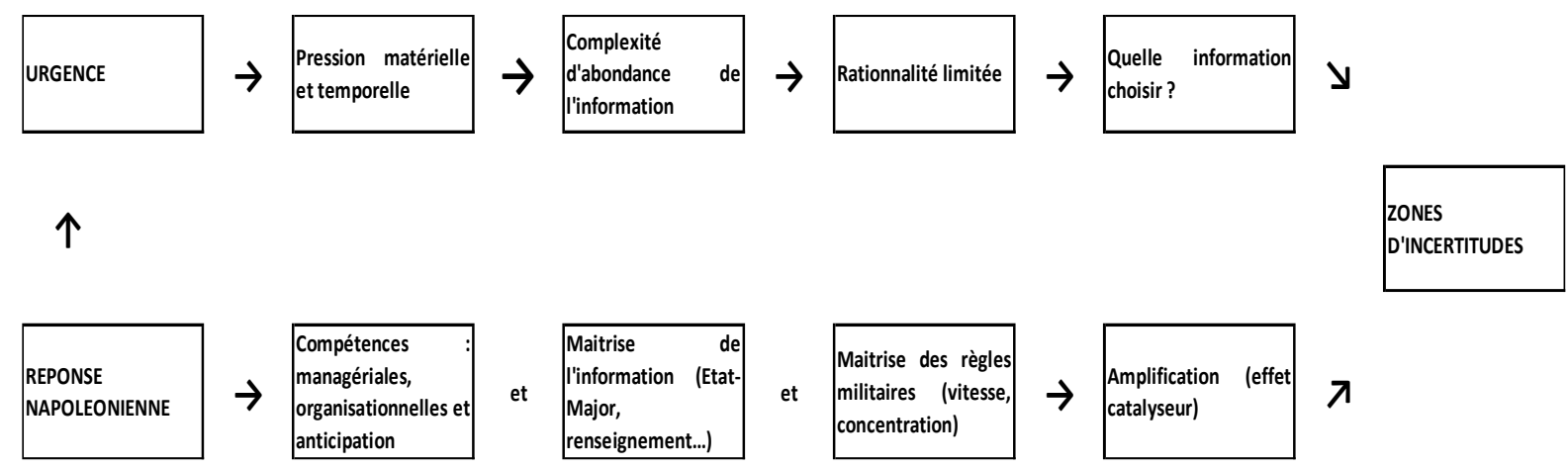

Mais encore une fois ce qui frappe chez Napoléon c'est son pragmatisme tant sur le plan politique que sur le plan militaire par l'utilisation de ses axiomes militaires préférés de vitesse et de mobilité. Avec lucidité, Napoléon sait très bien que lorsqu'il demande des déplacements rapides dans l'urgence, seuls certains d'entre eux tiendront l'objectif. Mais peu importe car ce qui compte pour Napoléon c'est qu'un minimum des soldats soit présent pour soutenir le choc comme s'il avait l'impression qu'il y a un effet de seuil permettant de tenir les assauts adverses en attendant l'arrivée des retardataires. Vitesse, mobilité et pragmatisme, assurent ainsi la concentration des forces.

En résumé, le management de Napoléon et son leadership lui permettent des attitudes proactives par lesquelles il gère en anticipant l'urgence pour ne pas subir les évènements. Pour cela, Napoléon dispose des facultés et des moyens organisationnels et communicationnels pour assurer la cohésion de ses troupes, facteur stratégique impératif pour mettre en application ses principes clés. Il le fera remarquablement en 1805 lors de la campagne d'Austerlitz ou en 1814 pour la campagne de France (défense du territoire national) où la concentration de ses forces lui permet de remporter des victoires dans un rapport de force pourtant défavorable d'un à huit. 
Par la suite, des troupes de plus en plus hétérogènes au niveau des nationalités rendront plus difficile le partage de valeurs communes, la cohésion des troupes et la concentration des forces. Mais les conditions de la réussite de Napoléon, face à l'urgence quasi-permanente à laquelle il fait face, s'observe avant tout par ses capacités à anticiper qui catalysent ses axiomes managériaux, ses moyens d'action et les résultats recherchés (figure 3). 
Figure 3. Le management de l'urgence par Napoléon Bonaparte (source : auteurs)

\begin{tabular}{|c|c|c|c|}
\hline \multirow[b]{2}{*}{$\begin{array}{c}\text { Formes de } \\
\text { management de } \\
\text { l'urgence }\end{array}$} & \\
\hline & Eléments & Caractéristiques & $\begin{array}{c}\text { Situations ou évènements } \\
\text { historiques les plus marquants }\end{array}$ \\
\hline \multirow{2}{*}{$\begin{array}{l}\text { PENSEES } \\
\text { AXIOMATIQUES }\end{array}$} & Leadership & $\begin{array}{l}\text { Charismatique, autoritaire, } \\
\text { paternaliste et persuasif }\end{array}$ & $\begin{array}{l}\text { Analyse de Weber (voir supra, Saint } \\
\text { Napoléon, Héroïsme, ...) } \\
\text { Typologie de Likert } \\
\text { Auteurs militaires de références }\end{array}$ \\
\hline & Principes (militaires) & $\begin{array}{l}\text { Rapidité } \\
\text { Mobilité }\end{array}$ & $\begin{array}{l}1^{\text {ère }} \text { et } 2^{\text {ème }} \text { campagne d'Italie, } \\
\text { Austerlitz } \\
\text { Friedland, Campagne de France }\end{array}$ \\
\hline \multirow[t]{2}{*}{$\begin{array}{l}\text { MOYENS } \\
\text { D'ACTION }\end{array}$} & Communication & $\begin{array}{l}\text { Communication } \\
\text { institutionnelle : emblèmes et } \\
\text { images } \\
\text { Communication interne : } \\
\text { formelle avec les proclamations } \\
\text { aux soldat, le rôle de l'Etat- } \\
\text { Major et informelles }\end{array}$ & $\begin{array}{l}1^{\text {ère }} \text { et } 2^{\text {ème }} \text { campagne d'Italie, camp de } \\
\text { Boulogne, rôle des emblèmes } \\
\text { Proclamations de la } 1^{\text {ère campagne }} \\
\text { d'Italie, d'Egypte, d'Austerlitz } \\
\text { Rôle de l'Etat-Major (Austerlitz, } \\
\text { Waterloo) }\end{array}$ \\
\hline & Organisation & $\begin{array}{l}\text { Au niveau de l'armée, structure } \\
\text { staff and line et structure par } \\
\text { projet. } \\
\text { Organisation flexible }\end{array}$ & $\begin{array}{l}\text { Armée de la } 2^{\text {ème }} \text { campagne d'Italie } \\
\text { Grande Armée } \\
\text { Chandler (1992) firme M } \\
\text { Multidivisionnelle }\end{array}$ \\
\hline \multirow[t]{2}{*}{ RESULTATS } & Cohésion & $\begin{array}{l}\text { Adhésion à des valeurs } \\
\text { communes (ascenseur social } \\
\text { pour les militaires, valeurs } \\
\text { républicaines) } \\
\text { Résultat de la communication et } \\
\text { de l'organisation }\end{array}$ & $\begin{array}{l}1^{\text {ère campagne d'Italie }} \\
\text { Campagne d'Egypte } \\
\text { Camps de Boulogne } \\
\text { Austerlitz, Iéna } \\
\text { Campagne de France (les «Marie } \\
\text { Louise ») }\end{array}$ \\
\hline & $\begin{array}{l}\text { Concentration } \\
\text { forces }\end{array}$ & $\begin{array}{l}\text { Concentration des forces } \\
\text { militaires par rapport à } \\
\text { l'adversaire malgré des effectifs } \\
\text { plus réduits } \\
\text { Résultat de la mobilité, de la } \\
\text { rapidité grâce à l'organisation } \\
\text { structurelle. }\end{array}$ & 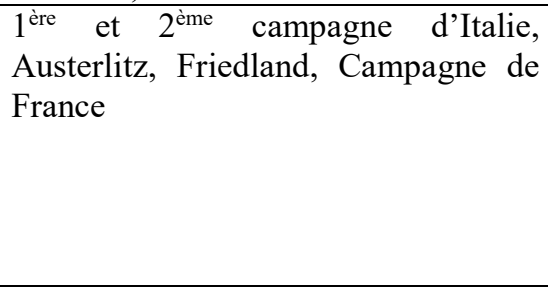 \\
\hline
\end{tabular}

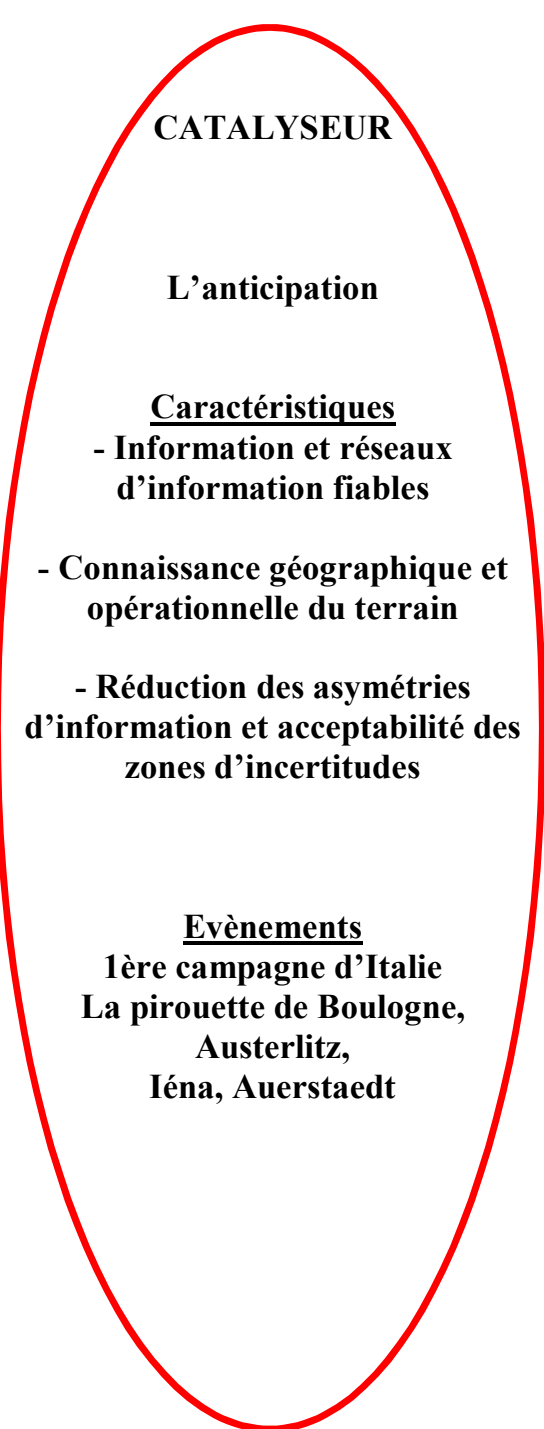




\section{LE CATALYSEUR DU MANAGEMENT DE L'URGENCE : L'ANTICIPATION}

Napoléon cherche en amont à anticiper les évènements, de manière rationnelle et informée, pour prendre les décisions les plus judicieuses y compris en situation d'urgence. Esprit pressé, Napoléon est donc bien dans le management quasi-permanent de l'urgence. Dans ce management, un élément clé, l'anticipation, catalyse tous les autres éléments.

D'après le Larousse, l'anticipation est «l'action de prévoir, d'imaginer des situations, des évènements futurs ». Par l'anticipation, l'individu se projette dans l'avenir en se représentant les résultats attendus d'une action cognitive ou de stratégies à mettre en œuvre pour y parvenir. Un catalyseur est un élément qui provoque une réaction par sa seule présence ou par son intervention (Larousse). Pour Napoléon Bonaparte, l'anticipation va permettre de mettre en application sa pensée managériale en provoquant l'action en termes de communication et d'organisation avec pour objectifs la cohésion et la concentration des forces.

Ainsi, l'anticipation permet-elle de gérer l'urgence en empêchant un scénario défavorable de se réaliser par la projection et la réalisation d'un autre scénario favorable.

A l'opposition rationalité limitée et création de zones d'incertitude -évoquée dans la revue de littérature- qui débouche sur des solutions différentes face à l'urgence, Napoléon, par ses anticipations, augmente la rationalité de ses stratégies tout en permettant l'usage des zones d'incertitudes créées, en termes d'autonomie et de responsabilité (figure 4). Cette anticipation s'appuie sur les moyens d'action, illustrées précédemment, que sont sa communication et son organisation militaire. Un exemple intéressant peut-être tiré de la comparaison des batailles d'Austerlitz en 1805 et d'Auerstaedt (concomitante de Iéna) en 1806 qui illustrent des modes opératoires d'anticipation très proches (figure 4). Ceci met en évidence, dans le management de l'urgence napoléonien, une articulation dynamique de modes d'action, pensés et activés de manière identique à des époques distinctes, et qui donne sa pleine mesure par l'effet catalyseur de l'anticipation. 
Figure 4. L'effet catalyseur de l'anticipation dans le management d'urgence (source : auteurs)

\begin{tabular}{|c|c|c|c|c|c|c|c|}
\hline $\begin{array}{c}\text { L'effet } \\
\text { catalyseur }\end{array}$ & $\begin{array}{c}\text { Les moyens } \\
\text { d'anticipation }\end{array}$ & $\rightarrow$ & $\begin{array}{c}\text { donnent un cadre } \\
\text { d'action } \\
\text { sécurisant et } \\
\text { réduisent les } \\
\text { asymétries } \\
\text { d'information }\end{array}$ & $\rightarrow$ & \begin{tabular}{|c|} 
augmentent la \\
rationalité en créant \\
des espaces de \\
liberté et \\
d'incertitude \\
acceptables et \\
maitrisables \\
\end{tabular} & $\rightarrow$ & $\begin{array}{c}\begin{array}{c}\text { Pour une } \\
\text { solution } \\
\text { performante et } \\
\text { innovante }\end{array} \\
\end{array}$ \\
\hline $\begin{array}{c}\text { Austerlitz } \\
\text { (1805) }\end{array}$ & $\begin{array}{l}\text { Organisation du } \\
\text { renseignement } \\
\text { général : } \\
\text { Communication } \\
\text { hiérarchique } \\
\text { et planification } \\
\text { des déplacements }\end{array}$ & $\rightarrow$ & $\begin{array}{c}\text { Connaissance du } \\
\text { terrain } \\
\text { Information sur les } \\
\text { troupes adverses et } \\
\text { leur } \\
\text { commandement }\end{array}$ & $\rightarrow$ & $\begin{array}{c}\text { Scénario et actions se } \\
\text { renforcent en termes } \\
\text { de probabilité }\end{array}$ & $\rightarrow$ & $\begin{array}{c}\text { Créativité dans } \\
\text { l'utilisation } \\
\text { inhabituelle des } \\
\text { hauteurs (Plateau } \\
\text { de Pratzen) et } \\
\text { victoire }\end{array}$ \\
\hline $\begin{array}{l}\text { Auerstaedt } \\
\text { (1806) }\end{array}$ & $\begin{array}{c}\text { Organisation de } \\
\text { la Grande Armée } \\
\text { (voir supra) }\end{array}$ & $\rightarrow$ & $\begin{array}{l}\text { Corps d'armée } \\
\text { dirigé par le } \\
\text { Maréchal Davout }\end{array}$ & $\rightarrow$ & $\begin{array}{c}\text { Utilisation flexible et } \\
\text { réactive des } \\
\text { Divisions Morand, } \\
\text { Gudin et Friant }(« \text { les } \\
3 \text { immortelles } »)\end{array}$ & $\rightarrow$ & \begin{tabular}{|c} 
à 2 contre 1 en \\
assumant seul - \\
sans l'aide de \\
Napoléon- ses \\
responsabilités, \\
le Maréchal \\
Davout remporte \\
la victoire.
\end{tabular} \\
\hline
\end{tabular}

L'anticipation napoléonienne semble un outil pragmatique pour concilier les différents types de solutions les plus performantes. Cependant, elle ne peut être efficace que si elle laisse un minimum de liberté aux subordonnés sur le terrain. Mais on ne peut tout prévoir et manager est un art qui mobilise chez lui une part intuitive et subjective propre à tout manager dans l'élaboration de stratégies. «Le grand art, c'est de changer pendant la bataille. Malheur au général qui arrive au combat avec un système. » (Napoléon Bonaparte). L'anticipation a donc aussi ses limites. L'exemple d'Auerstaedt est à la fois révélateur et paradoxal. En effet, à cette occasion, Napoléon n'a pas une information juste sur les mouvements ennemis et le même jour de l'affrontement d'Auerstaedt, il se bat victorieusement contre ces mêmes Prussiens à Iéna pensant combattre le gros de leur armée alors qu'il affronte en fait leur arrière garde.

Ainsi on peut distinguer deux types d'urgence aux frontières poreuses (figure 5) :

- L'urgence dans la durée où l'anticipation est stratégique mais aussi tactique et opérationnelle

- L'urgence dans l'instant où les principes, moyens définis grâce à l'anticipation dans la durée vont être les moyens d'y faire face d'un point de vue tactique et opérationnel mais aussi stratégique

L'anticipation étant une fonction décroissante du temps à l'opposé de l'urgence qui est croissante.

Dans les deux cas, l'anticipation en catalysant va créer des synergies faisant face à la chronologie de l'urgence. 
Figure 5. L'anticipation face à la chronologie de l'urgence

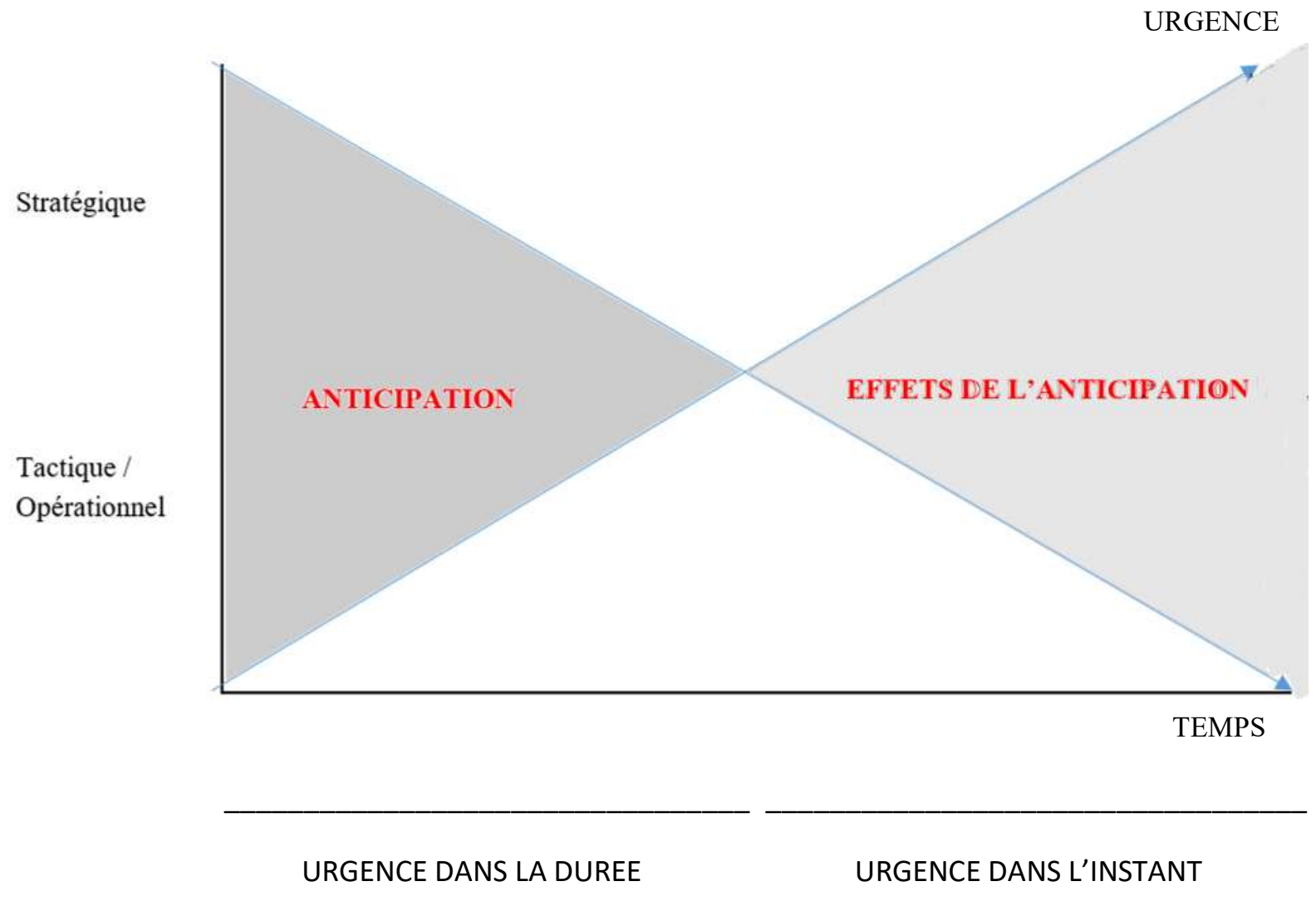

Dans le management de l'urgence par Napoléon, la communication et l'organisation structurelle et informationnelle apparaissent donc comme des atouts maîtres pour anticiper les situations d'urgence de manière performante. Lorsqu'ils sont absents, l'urgence peut déborder l'Empereur. En retour, l'anticipation construite catalyse la communication et l'organisation napoléonienne en l'associant à des principes militaires et un leadership constants et inébranlables créant des synergies. C'est cette dynamique crée par l'anticipation qui permis à Napoléon de construire la plupart de ces victoires militaires et son absence lui couta surement ses plus retentissants revers (Waterloo, campagne de Russie)

\section{CONCLUSION}

L'objectif de recherche de cet article était de mieux cerner le management napoléonien dans des situations d'urgence et de le rapprocher du management actuel des organisations. Les contributions scientifiques de cet article sont triples.

Tout d'abord, l'analyse des principales campagnes napoléoniennes, laisse entrevoir l'existence d'un mode de management de l'urgence par Napoléon guidé par une pensée managériale méconnue mais féconde, des moyens d'action adaptés à cette pensée et des objectifs destinés à répondre à l'urgence de manière quasi-permanente. 
Deuxièmement, la description de ce mode de management définit l'anticipation comme un principe dynamique et catalyseur du management de l'urgence napoléonien.

Enfin, nos analyses commencent à illustrer le caractère subjectif de l'urgence en management, que nous n'avons pas spécifiquement étudié ici, mais qui apparait de manière significative dans des évènements historiques étudiés. En effet, à la lecture des évènements militaires napoléoniens, il apparait que les situations d'urgence peuvent être contraintes comme créées. Napoléon, exprimant de manière magistrale, dans son mangement, cette capacité, d'une part, à faire face à l'urgence contrainte tout en la créant quand la situation l'impose.

En termes d'implication managériales, notre travail essaie de justifier l'intérêt d'essayer de reprendre, dans les organisations, des principes, très pratiques, appliquées par Napoléon dans son management de l'urgence. Au moins trois éléments de ce management nous semblent potentiellement reproductibles dans le monde de l'entreprise :

- la cohésion des hommes et des moyens par la communication et l'organisation staff and line,

- la concentration des forces sur un même point par des principes de mobilité et de vitesse.

- l'anticipation, d'une part, par la fiabilité de l'information et de ses réseaux et, d'autre part, par l'analyse et la connaissance permanente du terrain et des « forces » en présence.

Néanmoins, en décrivant les contributions théoriques et managériales de notre travail, nous avons essayé, en même temps, de suggérer leurs limites. Comme évoqué, plus haut, nos analyses ne peuvent pas prétendre à l'exhaustivité tant l'histoire napoléonienne est extrêmement riche en analyses et documents. A partir d'une sélection des principaux écrits les plus récents, dont les auteurs font autorité, nous avons principalement tenté de présenter les potentialités que présente, pour les sciences de gestion, l'analyse de son commandement et de son mangement face à une situation actuelle et nouvelle dans les organisations, telle que l'urgence. Par ailleurs, le caractère interprétatif de notre travail ne permet pas une généralisation de nos résultats et demandera sans doute de concevoir des travaux complémentaires sur la manière dont la pensée managériale de Napoléon peut impacter les principales fonctions de l'entreprise faisant face à l'urgence. Enfin, et parce que l'histoire napoléonienne peut être aussi controversée, nous avons tenté, comme évoqué plus haut, d'appréhender au mieux notre subjectivité afin d'éviter à la fois tout angélisme, mais aussi toute stigmatisation à l'égard de Napoléon et de son histoire militaire.

Napoléon parle lui-même de « prévoyance » que l'on peut assimiler à l'anticipation. Il s'agit ici de prévoir tout ce qui peut l'être et seulement ce qui peut l'être ce qui permet d'intégrer ainsi le hasard, appelé aussi « friction » par Napoléon et Clausewitz. « A la guerre, rien ne s'obtient que par calcul. Tout ce qui n'est pas profondément médité dans ses détails ne produit aucun 
résultat » (Napoléon à Joseph le 6 juin 1806). «Si je parais toujours prêt à répondre à tout, à faire face à tout, c'est qu'avant de rien entreprendre, j'ai longtemps médité, j'ai prévu ce qui pouvait arriver. ».

Cette anticipation, permet dans l'instantanéité à Napoléon d'avoir ce que Clausewitz appelle «le coup d'œil » dans l'urgence de la situation. Ce coup d'œil inclut toute cette réflexion effectuée à court, moyen mais aussi à long terme puisque joue pleinement ici les lectures par Napoléon des campagnes historiques des grands généraux. L'intuition, le $6^{\text {ème }}$ sens reconnus à Napoléon et plus largement aux bons managers ne seraient ainsi que le fruit de cette réflexion anticipatrice prenant appui sur des connaissances en histoire et comparant des situations analogues. Au-delà des présupposés bien souvent erronés, la qualité de celui qui commande est aussi de faire preuve d'humilité en cherchant des réponses auprès de ceux qui ont affrontés avec succès ces situations identiques. A l'instar de Napoléon, le manager doit anticiper l'urgence à la fois grâce au rationnel et au scientifique mais aussi à des niveaux plus intuitifs grâce à son expérience, d'autant qu'il est en situation de rationalité limitée et d'asymétrie d'information.

\section{BIBLIOGAPHIE}

AUBERT, N. (2003). Le culte de l'urgence: la société malade du temps (Vol. 2, p. 53). Paris: Flammarion.

AUBERT, N. (2006). L'urgence, symptôme de l'hypermodernité: de la quête de sens à la recherche de sensations. Communication et organisation, (29), 11-21.

BARZI, R. (2011). PME et agilité organisationnelle: étude exploratoire. Innovations, (2), 2945.

BERRY, M, MOISDON, JC, RIVELINE, C. (1979), Qu'est-ce que la recherche en gestion ?. Revue Informatique et Gestion, septembre-octobre.

BERRY, M. (1989) - La rationalité face à l'urgence. Gérer et Comprendre ${ }^{\circ} 17$,

BONARELLI, P. (1990), Rationalité et culture d'entreprise, thèse de doctorat en gestion de l'Ecole polytechnique,

BOUDON, J. O. (2000). Histoire du Consulat et de l'Empire: 1799-1815. Perrin.

BOUQUIN H. (2004), Le contrôle de gestion, Presses Universitaires de France, Collection Gestion, 6ème édition, Paris.

BOURGUIGNON A. (1995), Peut-on définir la performance ?, Revue Française de Comptabilité, juillet- août, pp. 61-66.

BOUSQUET, J. (2005). L'approche historique en marketing. Recherches qualitatives, 25(2).

BREU, K., HEMINGWAY, C. J., STRATHERN, M., BRIDGER, D., (2001), Workforce agility: the new employee strategy for the knowledge economy, Journal of Information Technology, 17, 21-31

BRUN, J-F (2008). Le cheval dans la Grande Armée. Revue historique des armées, 249 / 2008 BURNS, T ET STAKLER, G. (1994), The management of innovation, Oxford University Press. CANETTI, C., CATINAUD, C., \& KOCH, T. (2013). Manager l'urgence: Créer et gérer la rupture, diriger la transition. Dunod

CAO, J., ZHU, L., HAN, H., \& ZHU, X. (2018). Overview of Emergency Management. In Modern Emergency Management (pp. 3-11). Springer, Singapore. 
CASTELOT (1967). Bonaparte. Perrin.

CHANDLER, A.D. (1992) Organizational Capabilities and the Economic History of the Industrial Enterprise, Journal of Economic Perspectives 6(3): 79-100

COHEN M.D., JAMES C MARCH J.C, OLSEN, J.P (1972) «A garbage can model of organizational choice». Administrative Science Quarterly, vol. 17, p. 1 à 25.

COHENDET, P., LLERENA, P., 1999, Flexibilité et mode d'organisation, Revue Française de Gestion, (Mars-Mai), 72-7

COLIN, J. (1900). L'éducation militaire de Napoléon. Chapelot, Paris.

COLSON, B. (1999). L'Art de la guerre de Machiavel à Clausewitz, dans les collections de la Bibliothèque universitaire Moretus Plantin (9), Presses universitaires de Namur.

COLSON, B. (2011). De la guerre, Napoléon, présenté et annoté par Bruno COLSON. Perrin.

CROZIER, M., ET FRIEDBERG, E. (1977). L'acteur et le système. Seuil, Paris.

DE BOURCET, P. J. (1888). Principes de la guerre de Montagnes: 1775. Imprimerie Nationale.

DURHAM, L. N. L. (2015). The command and control of the grand armee: Napoleon as organizational designer. Pickle Partners Publishing

FIXARI, D., PALLEZ F. (1990) Petit traité d'urgence. Publication Ecole des Mines, octobre.

FIXARI, D., PALLEZ, F. (1992). Comment traiter l'urgence. Gérer et comprendre, (27), 7886.

GUIBERT, J-A (1772) Essai général de tactique. Economica

HADDOW, G., BULLOCK, J., \& COPPOLA, D. P. (2017). Introduction to emergency management. Butterworth-Heinemann.

JAKOBSON, R. (1960), Linguistics and poetics. In : Style in language. MA: MIT Press, 1960. p. $350-377$.

JAUREGUIBERRY, F. (2003). Les branchés du portable. Puf.

KALIKA, M. (1984), « Contribution à la connaissance de la structure organisationnelle: essai d'analyse systémique», Thèse de doctorat d'État en sciences de gestion, Bordeaux.

KATAYAMA, H., \& BENNETT, D. (1999). Agility, adaptability and leanness: a comparison of concepts and a study of practice. International Journal of Production Economics, 60, 43-51.

KIM D., CAVUSGIL S.T. ET CALANTONE R.J. (2006), Information System Innovations and Supply Chain Management: Channel Relationship and Firm Performance, 34(1), 40-54

KISH, L. (1982). Rensis Likert 1903-1981. The American Statistician, 36(2), 124-125.

LAÏDI Z., Le sacre du présent, Flammarion, Paris, 2000

LAWRENCE P.R. ET LORSCH J.W. (1973), Adapter les structures de l'entreprise. Les éditions d'organisation. 1973.

LIKERT, R.L. (1967), New Patterns of Management, McGraw-Hill Book Company, New York, NY.

LIKERT, R.L. (1974), Le gouvernement participatif de l'entreprise, Gauthier-Villars.

MATHEU, M. et ROQUEPLO, P. (1991), Urgences et raisons d'État, Gérer et Comprendre (23), juin.

MERIADE, L. (2016) Les simplifications instrumentales et processuelles: un dispositif de gestion des tensions de gouvernance publique à l'université. Revue française d'administration publique, 1/2016, 131-144.

MERIADE L. (2017), L'instrumentation des tensions de gouvernance publique (TGP) : L'exemple des simplifications des indicateurs de performance dans le système universitaire français, Management International 21(4).

MINTZBERG H. (1984), Le manager au quotidien. Les éditions d'organisation.

MINTZBERG, H. (2009). Managing. Berrett-Koehler Publishers. 
MINTZBERG, H. (2013). Simply managing: What managers do-and can do better. BerrettKoehler Publishers.

MIQUEL, P. (2005). Austerlitz. Albin Michel.

MOISDON J.C. (1990), Grands projets, organisation et urgence. Séminaire Vie des Affaires, 7 décembre.

MOREL, C. (2011), Les décisions absurdes: sociologie des erreurs radicales et persistantes T. III, Gallimard,

NOMBLOT, D., ET THOMAS, A. (2009), Il y a urgence à prendre le temps. La lettre de l'enfance et de l'adolescence, (2), 21-29.

PHILLIPS, B., NEAL, D. M., \& WEBB, G. (2016). Introduction to emergency management. CRC Press.

PIGEARD, A. (2000). L'armée de Napoléon. Organisation et vie quotidienne. Tallandier.

PIGEARD, A. (2004). Dictionnaire des batailles de Napoléon: 1796-1815. Tallandier.

PIOTET, F., \& SAINSAULIEU, R. (1994). Méthodes pour une sociologie de l'entreprise. Les Presses de Sciences Po.

POIREL, C., BONET FERNANDEZ, D., \& SERRANO, C. (2014). Responsiveness of a franchising network: a resource-based view (Working Paper, IPAG Business School No. 2014-250

PRINCE, S.H. (1920). Catastrophe and Social Change, Based Upon a Sociological Study of the Halifax Disaster. Ph.D. thesis. New York: Columbia University Department of Political Science.

REIX, R. (1975). La capacité de commande de la firme, thèse de doctorat, Université des sciences sociales de Grenoble).

REIX, R., 1989, La flexibilité, in Encyclopédie de Gestion, Paris, Economica, 1407-19.

RIVELINE, C. (1991). De l'urgence en gestion. Gérer et comprendre, 22, 82-92.

SAVITT, R. (1980). Historical Research in Marketing. Journal of Marketing, 44, 52-58

SAYLES, L. R. (1989). Leadership: Managing in real organizations. McGraw-Hill Book Company.

SEIFFERT, M. D., \& GODELIER, E. (2008). Histoire et gestion: vingt ans après. Revue française de gestion, (8), 17-30.

SHARIFI, H., ZHANG, Z. (1999), A methodology for achieving agility in manufacturing operations: an introduction, International Journal of Production Economics, 62 (1/2), 7-22.

SIMON H.A. (1957), Models of man social and rational, New York, Wiley.

SPRALLS S.A, HUNT S.D. ET WILCOX J.B. [2010], « Extranet Use and Building Reationship Capital in Interfirm Distribution Networks: The Role of Extranet Capability », Journal of retailing, Article in Press, Doi: 10.1016/j.retai.2010.09.001

SUCHET, A. (2004), Napoléon et le Management, Paris, Tallandier, in- $8^{\circ}, 264 \mathrm{p}$

TRANIE, J. (1999). L'épopée napoléonienne: les grandes batailles. Tallandier.

TULARD, J. (2012). Napoléon, chef de guerre. Tallandier.

WAUGH, W. L. (2015). Living with Hazards, Dealing with Disasters: An Introduction to Emergency Management: An Introduction to Emergency Management. Routledge.

WEBER, M. (1946). The sociology of charismatic authority. In H. H. Mills \& C. W. Mills (Eds. \& Trans), From Max Weber: Essays in sociology. New York: Oxford University Press.

WIEVIORKA, O.; DREVILLON, H.; HELARY, X., DERUELLE B.. GAINOT B. ; CREPIN A. (2018), Histoire militaire de la France, Edition Perrin, Ministère des armées

WOODWARD, J. (1980), Industrial Organization: Theory and Practice, Oxford University Press.

YUSUF, Y. Y., SARHADI, M., GUNASEKARAN, A. 1999, Agile manufacturing: the drivers, concepts and attributes, International Journal of Production Economics, 62 (1/2) 33-43. 